University of Nebraska - Lincoln

DigitalCommons@University of Nebraska - Lincoln

2010

\title{
Impact of woven fabric: Experiments and mesostructure-based continuum-level simulations
}

\author{
Ethan M. Parsons \\ Massachusetts Institute of Technology, emoparsons@gmail.com \\ Tusit Weerasooriya \\ US Army Research Laboratory \\ Sai Sarva \\ General Electric Global Research \\ Simona Socrate \\ Massachusetts Institute of Technology
}

Follow this and additional works at: https://digitalcommons.unl.edu/usarmyresearch

Part of the Operations Research, Systems Engineering and Industrial Engineering Commons

Parsons, Ethan M.; Weerasooriya, Tusit; Sarva, Sai; and Socrate, Simona, "Impact of woven fabric: Experiments and mesostructure-based continuum-level simulations" (2010). US Army Research. 143. https://digitalcommons.unl.edu/usarmyresearch/143

This Article is brought to you for free and open access by the U.S. Department of Defense at DigitalCommons@University of Nebraska - Lincoln. It has been accepted for inclusion in US Army Research by an authorized administrator of DigitalCommons@University of Nebraska - Lincoln. 


\title{
Impact of woven fabric: Experiments and mesostructure-based continuum-level simulations
}

\author{
Ethan M. Parsons ${ }^{\mathrm{a}, *}$, Tusit Weerasooriya ${ }^{\mathrm{b}}$, Sai Sarva ${ }^{\mathrm{c}}$, Simona Socrate ${ }^{\mathrm{a}}$ \\ a Massachusetts Institute of Technology, Cambridge, MA 02139, USA \\ ${ }^{\mathrm{b}}$ US Army Research Laboratory, Aberdeen Proving Ground, MD 21005-5069, USA \\ c General Electric Global Research, Niskayuna, NY 12309, USA
}

\section{A R T I C L E I N F O}

\section{Article history:}

Received 11 November 2009

Received in revised form

17 March 2010

Accepted 16 May 2010

\section{Keywords:}

Dynamics

Constitutive behavior

Finite elements

Impact testing

Woven fabric

\begin{abstract}
A B S T R A C T
Woven fabric is an increasingly important component of many defense and commercial systems, including deployable structures, restraint systems, numerous forms of protective armor, and a variety of structural applications where it serves as the reinforcement phase of composite materials. With the prevalence of these systems and the desire to explore new applications, a comprehensive, computationally efficient model for the deformation of woven fabrics is needed. However, modeling woven fabrics is difficult due, in particular, to the need to simulate the response both at the scale of the entire fabric and at the meso-level, the scale of the yarns that compose the weave. Here, we present finite elements for the simulation of the three-dimensional, high-rate deformation of woven fabric. We employ a continuum-level modeling technique that, through the use of an appropriate unit cell, captures the evolution of the mesostructure of the fabric without explicitly modeling every yarn. Displacement degrees of freedom and degrees of freedom representing the change in crimp amplitude of each yarn family fully determine the deformed geometry of the mesostructure of the fabric, which in turn provides, through the constitutive relations, the internal nodal forces. In order to verify the accuracy of the elements, instrumented ballistic impact experiments with projectile velocities of $22-550 \mathrm{~m} / \mathrm{s}$ were conducted on single layers of Kevlar ${ }^{\mathbb{R}}$ fabric. Simulations of the experiments demonstrate that the finite elements are capable of efficiently simulating large, complex structures.
\end{abstract}

(c) 2010 Elsevier Ltd. All rights reserved.

\section{Introduction}

Woven fabrics are used in a wide variety of defense and consumer products_both in neat form and as the reinforcement phase of composites. They are prized for their flexibility, formability, and high specific strength. Starting in the 1950s with ballistic Nylon ${ }^{\mathbb{R}}$ vests, personal body armor has been constructed from multiple plies of woven fabric for lightweight, flexible protection against bullets and fragmenting projectiles from sources such as grenades, mortars, mines, and improvised explosive devices. Woven fabrics also reinforce polymers or ceramics in helmets and armor panels in order to defeat high velocity projectiles. Additional common applications include restraint systems such as seat belts and head restraints, deployable fabric structures such as air bags, air beams, and parachutes, as well as other forms of ballistic protection such as spall liners, bomb blankets, and turbine engine fragment barriers. Recently, woven fabrics have been integrated with flexible electronics, microfluidics, and actuated materials to form "smart textiles."

\footnotetext{
* Corresponding author. Tel.: +1 6175012968.

E-mail address: emoparsons@gmail.com (E.M. Parsons).
} 
Despite the widespread use of woven fabrics, there is no comprehensive, universally accepted computational model for the deformation of woven fabrics. The design of woven fabric systems is still largely based on experience and empirical methods. As such, the analysis of existing systems and the development of new applications is overly costly and time consuming.

Modeling woven fabrics is challenging due in part to the complexity of the weave architecture. At the mesoscale, a woven fabric consists of two orthogonal sets of yarns interlaced with one another. In a ballistic application, each yarn is typically made up of hundreds of highly oriented continuous polymer fibers. During the weaving process, the parallel warp yarns are held taut in the loom, aligned with the finished edge of the fabric. The neddles of the loom move the warp yarns up and down, allowing the shuttle to carry the fill or weft yarns through the gaps. When the fabric is released from the loom, neither yarn family lies flat in the plane of the fabric. The resulting undulation of each yarn over and under the orthogonal yarns is described as crimp, and wherever the yarns cross is termed a cross-over point. The crimp amplitude is the wave amplitude of the yarn. The macroscopic response of the fabric is highly dependent on the current orientation and crimp of the yarns.

The second reason that modeling woven fabrics is difficult is the variety of deformation mechanisms available to the yarns. The in-plane deformation of woven fabrics may be grouped into five major modes:

1. Yarn stretch: The elongation of the yarns, a stiff mode of deformation, may be approximately linear and rateindependent $\left(\operatorname{Kevlar}^{\mathbb{R}}\right.$ ) or decidedly viscoelastic (Nylon).

2. Uncrimping: Depending on the type of loading, the initially undulating yarns straighten via two different modes of deformation. Uniaxial tension parallel to a yarn direction, for example, causes crimp interchange to occur: the loaded yarns straighten while the yarns of the other family become more crimped. Crimp interchange can occur with very little stretch of the yarns, resulting in a compliant response until the yarns under load straighten. On the other hand, equibiaxial tension parallel to the yarn directions, for example, causes uncrimping in both yarn families via transverse compression of the yarns at the cross-over points. There is at first little resistance to transverse compression due to the voids between the fibers in each yarn. Transverse compression is thus another source of an initially compliant response. At high rates of loading, the inertia of the yarns also plays a significant role in uncrimping.

3. Relative yarn rotation or trellising: The change in in-plane angle between the two yarn families, trellising has both an elastic component, due to rotation between cross-over points, and a dissipative component, due to the frictional resistance to rotation at the cross-over points. Frequently termed "shear," trellising is the primary mode by which large deformations are accommodated in woven fabrics.

4. Locking: The resistance to trellising increases as yarns of opposite families shear-lock against one another in the plane of the fabric. Cross-locking occurs when crimp interchange causes lateral contraction of the fabric to an extent that the loaded yarns jam against one another.

5. Yarn slip: The relative displacement of one yarn family with respect to the other at the cross-over points, yarn slip permanently alters the mesostructure of the fabric and occurs with little resistance under certain circumstances. In this document, the fabric is approximated as slip-free.

These five meso-level modes of deformation combine to create the nonlinear, anisotropic response of woven fabrics to arbitrary loadings. In short, woven fabrics are quite stiff in the yarn directions, shear easily up to a certain angle, and generally exhibit a delayed stress response due to uncrimping of the yarns.

A comprehensive model for woven fabrics must track the evolving geometry of the mesostructure as well as account for all of the major meso-level modes of deformation. A faithful representation of the geometry and modes of deformation is essential for the accurate prediction of the macroscopic response of the fabric. Furthermore, the yarn-level aspects of deformation underlying the macroscopic response are important in their own right: yarn tension or strain is the criterion for failure due to projectile impact. Yarn spacing or areal density determines the susceptibility of the fabric to puncture threats and chemical agents. In composite forming operations, excessive yarn spacings and shear angles cause defects in the formed parts while yarn spacing, orientation, and crimp amplitude determine the mechanical properties of the formed parts. Large changes in crimp amplitude and high contact forces between yarns may break or damage interwoven components in smart textiles.

The majority of the models in the literature suitable for simulating general fabric deformations take one of two finite element approaches: either they discretely model in three dimensions every yarn (or even fiber) in the weave or they homogenize the response of the yarns, typically by treating the fabric as an anisotropic continuum with properties determined from experiments or micromechanical modeling. The discrete, three-dimensional approach, used by Shockey et al. (1999), Duan et al. (2006b), Zhang et al. (2008), and Talebi et al. (2009), among others, is capable of accurately representing both the geometry of the fabric and the interactions between yarns. It is thus valuable for studying the various modes of deformation of the yarns and the effects of parameters such as weave style and yarn properties on macroscopic deformation. However, building these elaborate finite element meshes is tedious, and simulating actual structures or events of significant duration is computationally prohibitive in most cases. In addition, integration of discrete yarn models with other material models for the simulation of composites is complicated. At the opposite end of the spectrum, the homogenized, continuum approach, used by Lim et al. (2003), Xue et al. (2003), and Peng and Cao (2005), 
among others, is computationally efficient and readily combined with other material models. Although this approach can accurately represent the macroscopic response of woven fabrics, it does not explicitly model the modes of deformation or interactions of the yarns, and therefore cannot predict all of the necessary yarn-level aspects of deformation. Another drawback of the traditional continuum approach is that the homogenized material properties are not explicit functions of physical yarn parameters. Thus, parametric studies or the design of new fabrics cannot be undertaken simply by changing the geometry of the weave or the properties of the yarns.

There are also a number of models specific to the ballistic impact of woven fabrics. Roylance et al. (1973) modeled the fabric as a planar array of point masses connected by massless, pin-joined trusses. The model of Roylance et al. has been extended to account for nonlinear viscoelastic yarn behavior (Roylance and Wang, 1978; Shim et al., 1995), yarn slip (Roylance et al., 1995; Termonia, 2004; Tan and Ching, 2006), and crimp interchange (Ting et al., 1998; Tan et al., 2005). However, none of these models includes all of the major meso-level modes of deformation. Furthermore, with this type of model, it is difficult to include the contact between layers in multi-ply simulations and to incorporate the matrix material in the simulation of fabric-reinforced composites. Other investigators, including Taylor and Vinson (1990), PargaLanda and Hernández-Olivares (1995), Cunniff (1996), and Phoenix and Porwal (2003), have proposed useful analytic models for ballistic impact of woven fabrics, but these models cannot simulate arbitrary geometries, loadings, and boundary conditions.

In this work, we used a continuum-level modeling technique for woven fabrics that bridges the gap between the discrete, three-dimensional approach and the traditional continuum-level approach. With this technique, termed by some the semi-discrete or meso-macro approach, at each integration point in the finite element model, the homogenized continuum properties are determined from a deforming analytic model of the mesostructure of the fabric. The analytic model is a three-dimensional representation, constructed of pin-joined trusses, of the unit cell of the fabric. It is similar to the analytic model of Kawabata et al. (1973) but, following King et al. (2005), we add locking trusses and a cross-over spring connecting the yarns. The deformed geometry of the fabric unit cell is determined by the macroscopic deformation gradient and the satisfaction of transverse equilibrium at the cross-over point. The forces on the unit cell, and in turn the corresponding continuum-level stresses, are calculated from physically motivated constitutive relations. This technique captures the macroscopic response of the fabric as well as the evolution of its mesostructure with deformation in a single efficient step. Together, the unit cell geometry and the constitutive relations accurately represent yarn stretch, crimp interchange, trellising, and locking. As such, discrete yarn properties and interactions are simulated with the computational efficiency and versatility of the continuum-level approach. Although not included in this document, yarn slip is also readily modeled with this technique. When yarn slip is included, the fabric continuum is described not by the location of the yarns but by the location of the cross-over points. King (2006) presented a quasi-static constitutive model with yarn slip, and a finite element suitable for dynamic applications is in development.

Starting with the planar constitutive model of King et al., we have developed six-node and nine-node finite elements for the high-rate, three-dimensional deformation of woven fabrics. King et al. implemented their constitutive model into traditional Abaqus/Standard finite elements for the simulation of quasi-static extension and shear tests. They solved for the deformed geometry of the fabric unit cell by minimizing the elastic energy stored in the unit cell via an iterative numerical scheme. Here, we construct our own membrane finite elements and define, with appropriate mass, not only threedimensional displacement degrees of freedom, but also degrees of freedom to represent the change in crimp amplitude of each yarn family. We use these amplitude degrees of freedom, together with transverse equilibrium, to solve directly for the deformed geometry of the unit cell, a method which reduces simulation times by several hundred percent when compared with the energy minimization method. Specifying the changes in crimp amplitude as actual degrees of freedom has several other advantages: First, the degree of freedom approach enables us to use the correct transverse compression properties of the yarns. With the energy approach, transverse compression of the yarns must be modeled as artificially stiff in order to prevent the yarns from passing through one another. Second, the degree of freedom approach is more accurate than the energy approach because, with the energy approach, the energy is minimized at each individual unit cell, and, as Grujicic et al. (2008b) noted, the configuration of the fabric may therefore not correspond to a global energy minimum. Finally, with the degree of freedom approach, the inertia of uncrimping is physically represented by the mass associated with each crimp degree of freedom.

A number of other authors have presented models for woven fabrics with continuum properties calculated from a deforming unit cell. Boisse et al. $(1997,2001)$ constructed a three-dimensional unit cell like that of Kawabata for quasistatic and dynamic simulations of composite forming but did not incorporate the resistance to yarn rotation or the inertia of uncrimping. For similar dynamic simulations, Boisse et al. $(2005,2006)$ later used a planar unit cell which incorporated shear resistance at the cross-over point but did not include cross-locking. Hamila et al. (2009) extended this approach to a shell finite element which included the resistance to bending of the yarns. Boisse et al. (2005, 2006) and Hamila et al. simulated the effect of uncrimping on the tensile response of the fabric by determining yarn tension surfaces, $T\left(\varepsilon_{11}, \varepsilon_{22}\right)$, from the results of biaxial tensile tests at different stress ratios. This approach, unlike that of the finite elements presented in this document, does not explicitly simulate the change in crimp amplitude of the yarns and therefore requires more experiments and fitting of the constitutive relations than our approach. Tanov and Brueggert (2003) employed a planar unit cell with locking trusses in order to capture yarn rotation and shear-locking in the simulation of air bags but did not incorporate uncrimping. Ivanov and Tabiei (2004) constructed a Kawabata type unit cell for the simulation of ballistic impact but did not consider transverse compression, cross-locking, or the inertia of uncrimping. Shahkarami and Vaziri 
(2007) also used the Kawabata geometry in the simulation of ballistic impact but did not include cross-locking, the inertia of uncrimping, or dissipative yarn rotation. Boisse et al. (1997, 2001), Ivanov and Tabiei, and Shahkarami and Vaziri all determined the crimp amplitudes of the yarns via time-consuming iterative numerical schemes.

Grujicic et al. (2008b) added yarn failure to the constitutive model of King et al. (2005) and King (2006) and implemented it as a material subroutine in a dynamic finite element scheme. The ballistic impact of a fabric composed of Kevlar 129 yarns was simulated with shell elements and compared with simulations with each yarn modeled discretely. Values from the literature were used for the fabric geometry, yarn stiffness in tension, and the theoretical calculation of the yarn bending stiffness, but all other material parameters, including those for yarn slip and out-of-plane deformation, were identical to those determined by King for Kevlar S706 with KM2 yarns. Unlike the work presented in this document, Grujicic et al.'s implementation used the energy minimization procedure of King et al. to calculate the deformed geometry of the fabric unit cell and modeled the resistance of the yarns to transverse compression as overly stiff. Grujicic et al. did not explicitly model the inertia of uncrimping and also omitted the rate dependence of yarn rotation.

The rest of this document is organized as follows: Section 2 presents the woven fabric model and finite elements in further detail, specific in parts to the plain-weave fabric Kevlar S706. The geometry of the unit cell is given, and constitutive relations are introduced to describe the major modes of deformation of woven fabrics. This section also explains how the deformed geometry of the unit cell and, in turn, the continuum-level stresses in the fabric are determined. Section 2 concludes with the specifics of the fabric finite elements and successful comparisons of simulations with simple quasi-static experiments. Section 3 describes the low velocity projectile impact experiments performed at the US Army Research Laboratory and the simulations thereof. In these experiments, the displacements everywhere on the fabric were provided by digital image correlation. The simulations of the experiments show the elements to be accurate at higher rates and more complex loadings than those of the quasi-static experiments of Section 2 . Section 4 describes the ballistic impact experiments performed at the MIT Institute for Soldier Nanotechnologies and the simulations thereof. The ability of the elements to predict deformation and failure at very high strain rates is demonstrated. Section 5 , the conclusion of the document, highlights the success of the elements and provides examples of potential applications and extensions.

\section{Woven fabric model and finite elements}

At an appropriate scale, sufficiently larger than the spacing of the yarns, woven fabric behaves homogeneously and thus can be approximated as a continuum. We model the deformation of a section of discrete yarns with a single material point using the technique depicted schematically in Fig. 1. Each discrete yarn has its own tension and shear force. By the continuum assumption, the deformation of the section of discrete yarns can be represented by the deformation of an appropriate unit cell. The deformed geometry of the unit cell is determined by the deformation gradient and transverse equilibrium at the material point. From the deformed geometry, constitutive relations describing the major modes of deformation of woven fabrics determine the tensile and shear forces on the unit cell. The forces are then homogenized over the boundary of the unit cell in order to calculate a continuum-level stress measure for the section of fabric. The continuum-level stress is used to calculate the internal nodal forces required by the finite elements.

The constitutive relations were developed and calibrated by King et al. (2005) through simulations and experiments on Kevlar $^{\mathbb{R}}$ S706, a plain-weave fabric composed of 600 denier Kevlar KM2 yarns at a pitch of 34 threads per inch. KM2 yarns

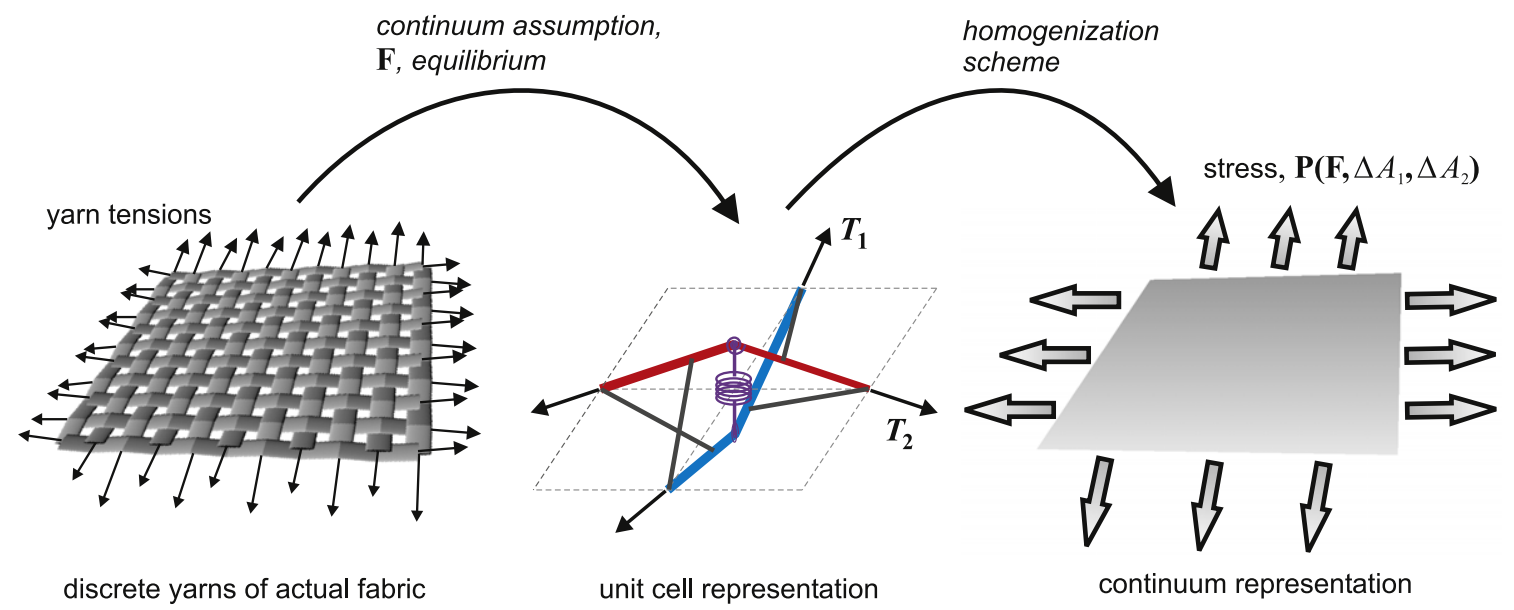

Fig. 1. Schematic of continuum modeling technique for woven fabric: discrete yarn tensions are transformed into continuum-level stresses via the deformation of a unit cell. Shear forces are omitted from the schematic for clarity. 
are untwisted and consist of 400 para-aramid fibers, each $6 \mu \mathrm{m}$ in diameter. The modeling technique is general, however, and could straightforwardly be applied to other plain-weave fabrics. Fabrics of different weave patterns would require more complex unit cells, but the form of the constitutive relations would remain the same.

We have developed finite elements for woven fabrics with degrees of freedom representing the macroscopic displacement of the fabric and the change in crimp amplitude of each yarn family. We have implemented these elements into the dynamic finite element code Abaqus/Explicit with the user element subroutine. This implementation can be used to simulate a variety of structures under loads of arbitrary geometry and rate. In this section, we demonstrate the accuracy of the elements at low strain rates by presenting simulations of actual strips of fabric loaded in uniaxial tension.

\subsection{Unit cell of plain-weave fabric}

The backbone of the model is the assumed geometry of the unit cell of the fabric. The unit cell approximates the mesoscale modes of deformation that underlie the macroscopic response of the fabric. The unit cell of a plain-weave fabric consists of two crossing sections of yarn (Fig. 2). Due to the symmetry of the structure, only one-quarter of the actual repeating unit needs to be considered. The warp $(k=1)$ and weft $(k=2)$ yarns are each approximated as pin-joined, elastic trusses with the following geometric properties (Fig. 2a):

(1) yarn quarter-wavelength or half-spacing, $p_{k}$;

(2) yarn half-length or simply "length," $L_{k}$;

(3) yarn crimp angle, $\beta_{k}$;

(4) yarn crimp amplitude, $A_{k}$;

(5) in-plane angle between yarns, $\theta$.

The initial values of these properties were taken from measurements of the fabric (Table 1). These properties are not independent, for any two of $p_{k}, L_{k}, \beta_{k}$, and $A_{k}$ are sufficient to determine the geometry of yarn $k$. The trusses are extensible but infinitely stiff in bending. The resistance to bending of the yarns is lumped in torsional bending springs at the pin-joints. A cross-over spring connecting the yarns provides torsional resistance to yarn rotation as well as compressive
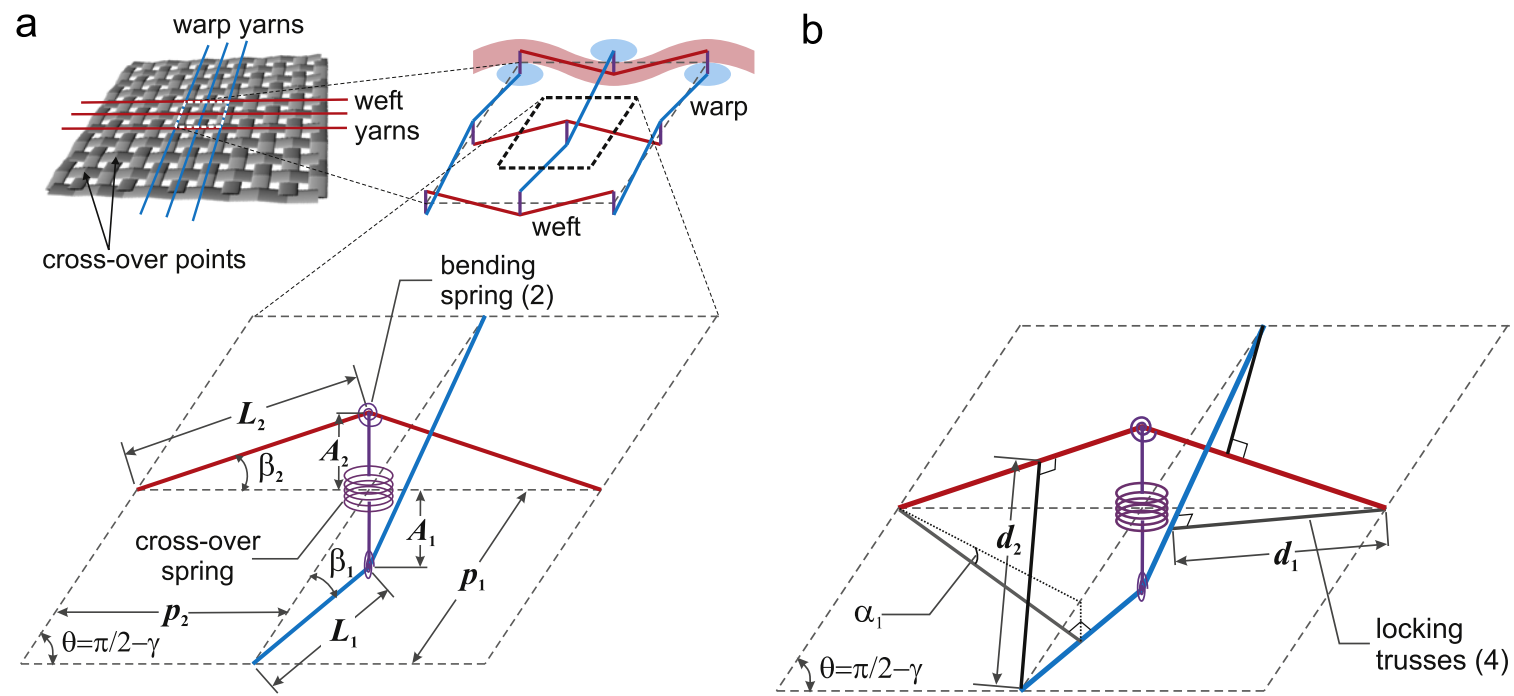

Fig. 2. Unit cell of plain-weave fabric: (a) geometry of warp and weft yarns, (b) locking trusses to simulate shear-locking and cross-locking of the fabric.

Table 1

Initial geometric properties of Kevlar S706.

Warp quarter-wavelength, $p_{1}^{0}$

Weft quarter-wavelength, $p_{2}^{0}$

Warp crimp amplitude, $A_{1}^{0}$

Weft crimp amplitude, $A_{2}^{0}$

In-plane angle, $\theta^{0}$
$0.374 \mathrm{~mm}$

$0.374 \mathrm{~mm}$

$0.060 \mathrm{~mm}$

$0.090 \mathrm{~mm}$

$90^{\circ}$ 
resistance to compaction of the yarns. Locking trusses resist both shear-locking and cross-locking of the yarns. Each locking truss extends from a boundary of the unit cell to a perpendicular point on a yarn and has geometric properties (Fig. 2b):

(1) locking truss length, $d_{k}$;

(2) angle between locking truss and plane of the fabric, $\alpha_{k}$.

\subsection{Woven fabric constitutive relations}

The five major in-plane, yarn-level modes of deformation, yarn stretch, crimp interchange, yarn rotation, locking, and yarn slip, are described by constitutive relations (with superscript "0" indicating an initial, undeformed value). These constitutive relations were determined by King et al. (2005) from experiments on Kevlar S706. All material parameters used in this work, except those for transverse compression of the yarns, were taken from King et al.

\subsubsection{Yarn stretch}

The stiffnesses of the yarns were measured with tensile tests on single yarns carefully pulled from the fabric. Once uncrimped, KM2 yarns have been found to be linear elastic and virtually rate-independent up to strain rates of at least 2500/s (Cheng et al., 2005), and the yarn tensions are therefore given by

$$
T_{k}=k_{k}\left(L_{k}-L_{k}^{0}\right) \text {, }
$$

where $k_{k}, k=1,2$ is the stiffness of the warp yarns and weft yarns, respectively. The warp and weft stiffnesses differ slightly due to damage incurred during the weaving process.

\subsubsection{Uncrimping}

Uncrimping, typically through a combination of crimp interchange and transverse compression of the yarns, is a major source of nonlinear deformation and anisotropy in woven fabrics. It is controlled by the resistances to bending and transverse compression of the yarns. The resistances to bending are very small due to the ease by which the many fibers in each yarn slide by one another. Fit to the uncrimping portion of the single yarn tests, the bending responses were approximated as linear elastic, resulting in bending moments,

$$
M_{\mathrm{b} k}=k_{\mathrm{b} k}\left(\beta_{k}-\beta_{k}^{0}\right) \quad(k=1,2),
$$

in the torsional bending springs at the pin-joints. If the fabric is stretched biaxially, the change in crimp amplitude of the yarns is largely accommodated by transverse compression or "interference" of the yarns. The resistance to transverse compression was measured by compressing a stack of fabric specimens in the through-thickness direction. The compressive force in the cross-over spring, termed the interference force, is given by the exponential expression,

$$
F_{\mathrm{I}}=K_{\mathrm{I}}\left(\mathrm{e}^{a \mathrm{I}}-1\right) \text {, }
$$

where $I=-\Delta A_{1}-\Delta A_{2}$ is the interference of the yarns at the cross-over point. King et al. scaled the compressive component of the cross-over spring in order to prevent the yarns from passing through one another. In this work, we fit Eq. (3) to the

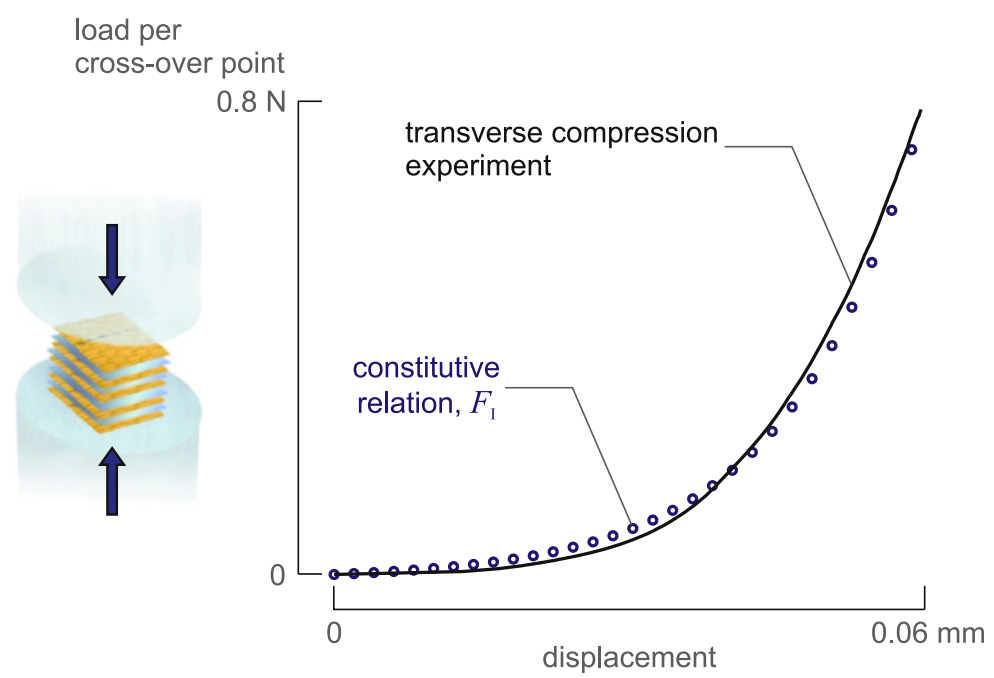

Fig. 3. Fit of the constitutive relation for interference force, $F_{\mathrm{I}}$, to the transverse compression experiment (King et al., 2005). 
actual compression tests of King et al., finding $K_{\mathrm{I}}=9.0 \times 10^{-3} \mathrm{~N}$ and $a=7.5 \times 10^{4} \mathrm{~m}^{-1}$ (Fig. 3). There is evidence that the resistance to transverse compression is a function also of the tensions in the yarns (Realff et al., 1997; Boisse et al., 2001). With additional experiments, Eq. (3) could be modified to include this effect.

\subsubsection{Yarn rotation}

Large deformations of woven fabrics may be accommodated by relative yarn rotation, $\gamma$, also known as trellising or in-plane shear. Cao et al. (2008) demonstrated that the resistance to yarn rotation is well characterized by the shear-frame experiment, a test in which a square specimen is clamped in a pin-joined "picture-frame" and stretched from two opposite corners. King et al. performed shear frame experiments at several different strain rates. During these tests, the fabric has an initial stiff, elastic response due to rotation of the yarns between the cross-over points,

$$
M_{\mathrm{s}}=K_{\mathrm{s}} \gamma^{\mathrm{e}} \text {, }
$$

followed by a compliant, rate-dependent, dissipative response due to rotation at the cross-over points under the effect of friction,

$$
\dot{\gamma}_{\mathrm{f}}=\dot{\gamma}_{0}\left(\frac{M_{\mathrm{s}}}{M_{0}}\right)
$$

resulting in a total relative yarn rotation, $\gamma=\gamma^{\mathrm{e}}+\gamma^{\mathrm{f}}$ (Fig. 4a). Fit to the results of shear-frame experiments typified by the response in Fig. $4 \mathrm{~b}$, these relations are represented in the unit cell by the torsional resistance of the cross-over spring. $K_{\mathrm{s}}$ was determined from the initial slope of the plot in Fig. $4 \mathrm{~b}$, while $\dot{\gamma}_{0}$ and $M_{0}$ were determined from the forces at which dissipative rotation began at several strain rates.

\subsubsection{Locking}

At some point, warp and weft yarns contact one another in the plane of the fabric, and further yarn rotation is also resisted by cross-sectional compaction of the yarns. Termed shear-locking, this resistance is provided by the compressive force in the locking trusses:

$$
F_{\mathrm{L} k}=\left\{\begin{array}{ll}
0, & d_{k}^{0}-d_{k} \leq 0 \\
K_{d}\left(d_{k}^{0}-d_{k}\right)^{\mathrm{c}}, & d_{k}^{0}-d_{k}>0
\end{array} \quad(k=1,2) .\right.
$$

The locking trusses also provide resistance to cross-locking, the jamming of yarns of the same family against one another. They have stiffness in compression only, and their properties, $K_{d}$ and $c$, were also determined from the shear-frame experiment (Fig. 4b). $K_{d}$ was fit to the approximately constant slope of the dissipative part of the response, and $c$ was chosen such that locking, indicated by the increase in slope of the response, begins at the appropriate shear angle.

\subsubsection{Yarn slip}

Large deformations of woven fabrics may also be accommodated by relative yarn slip at the cross-over points. Driven by gradients in yarn tension, yarn slip permanently alters the mesostructure of the fabric. It occurs, for example, at the unconstrained edges of a fabric, where it is manifested as unraveling, and at the ends of a slit. In this work, we pin the yarns at the cross-over points, disabling slip, primarily because Kevlar S706 is a tightly woven fabric. The yarns are jammed against one another even in the unloaded state, creating a large frictional resistance to slip. Yarn slip will be incorporated into the fabric finite elements in a future publication.
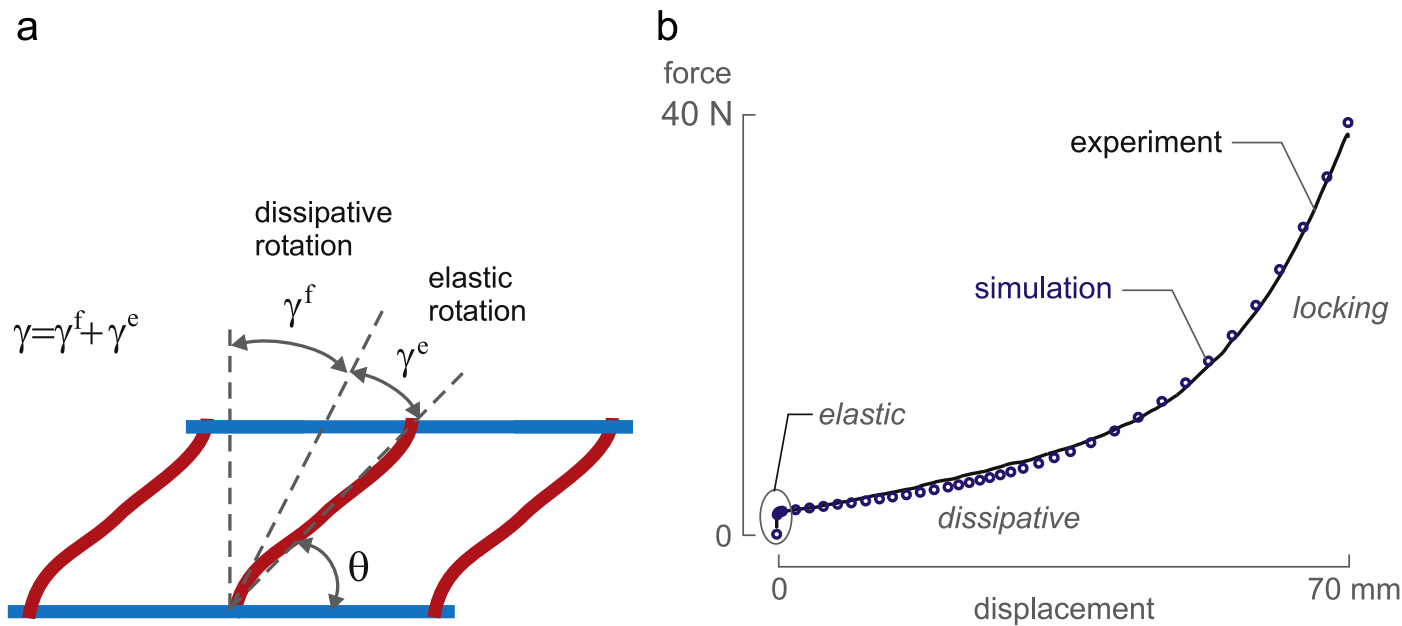

Fig. 4. The resistance to relative yarn rotation has elastic, dissipative, and locking components: (a) schematic of types of yarn rotation, (b) fit of model to a shear frame experiment with $\dot{\gamma} \approx 2.8 \times 10^{-3} \mathrm{rad} / \mathrm{s}$ (King et al., 2005). 


\subsection{Deformed geometry of the fabric unit cell}

The deformed geometry of the unit cell in three dimensions is fully determined by the macroscopic deformation gradient, $\mathbf{F}=\operatorname{Grad} \mathbf{x}(\mathbf{X}, t)$, and transverse equilibrium. At the continuum level, at each material point in the undeformed configuration, the fabric is described by unit vectors $\mathbf{g}_{1}^{0}$ and $\mathbf{g}_{2}^{0}$, aligned with the warp yarns and weft yarns, respectively. These yarn vectors are material lines, and the deformation gradient specifies their orientation and length everywhere on the deformed fabric:

$$
\mathbf{g}_{1}=\mathbf{F g}_{1}^{0} \quad \text { and } \quad \mathbf{g}_{2}=\mathbf{F g}_{2}^{0}
$$

The angle between the yarns is

$$
\theta=\cos ^{-1}\left(\frac{\mathbf{g}_{1} \cdot \mathbf{g}_{2}}{\left|\mathbf{g}_{1}\right|\left|\mathbf{g}_{2}\right|}\right)
$$

and the stretch of the yarn vectors is simply

$$
\lambda_{1}=\left|\mathbf{g}_{1}\right| \text { and } \lambda_{2}=\left|\mathbf{g}_{2}\right|,
$$

which enables the calculation of the current yarn quarter-wavelengths,

$$
p_{1}=\lambda_{1} p_{1}^{0} \quad \text { and } \quad p_{2}=\lambda_{2} p_{2}^{0}
$$

Although $p_{k}$ is specified, the yarn length, $L_{k}$, and crimp amplitude, $A_{k}$, may still take any of an infinite number of combinations. One or the other must be solved for in order to fully determine the deformed geometry of the unit cell. The out-of-plane state of the fabric is thus calculated from a force balance over each yarn (Fig. 5). At the end of each cross-over spring, for static equilibrium, the force in the cross-over spring, $F_{\mathrm{I}}$, must balance the net transverse component of the force in the yarn trusses, $F_{k}^{\mathrm{N}}$ :

$$
F_{\mathrm{I}}=2 F_{k}^{\mathrm{N}} \quad(k=1,2) .
$$

$F_{\mathrm{I}}$ is given by Eq. (3), and $F_{k}^{\mathrm{N}}$ depends on the moments in the bending springs and the forces in the yarns and locking trusses:

$$
F_{k}^{\mathrm{N}}=T_{k} \sin \beta_{k}-F_{\mathrm{L} l} \sin \alpha_{l}\left(1+\frac{p_{l}|\cos \theta| \cos \beta_{k}}{L_{k}}\right)+\left(\frac{k_{\mathrm{b} k}\left(\beta_{k}-\beta_{k}^{0}\right)}{L_{k}}\right) \cos \beta_{k},
$$

where the geometric properties of the fabric are defined in Section 2.1 and $l=1,2(l \neq k)$. In the next section, we use Eq. (11) to solve for the changes in crimp amplitude, $\Delta A_{1}$ and $\Delta A_{2}$.

\subsection{Calculating fabric stresses from yarn forces}

The continuum response at each material point on the fabric is obtained through homogenization of the unit cell response. Once the deformed geometry of the unit cell is known, the forces in the yarns are determined from the constitutive relations. In order to convert the yarn forces into a continuum-level stress measure, the stress on the boundary of the unit cell is computed. The first Piola-Kirchoff stress, $\mathbf{P}=\mathbf{P}\left(\mathbf{F}, \Delta A_{1}, \Delta A_{2}\right)$, is calculated by equating the external stress which must act on the loaded unit cell with the in-plane forces that the yarns exert on the unit cell. Fig. 6 shows the forces due to the tension in the yarns, $T_{k}$, and the moment in the cross-over spring, $M_{\mathrm{s}}=2 F_{k}^{\mathrm{Ms}} p_{k}$. These forces are projected onto

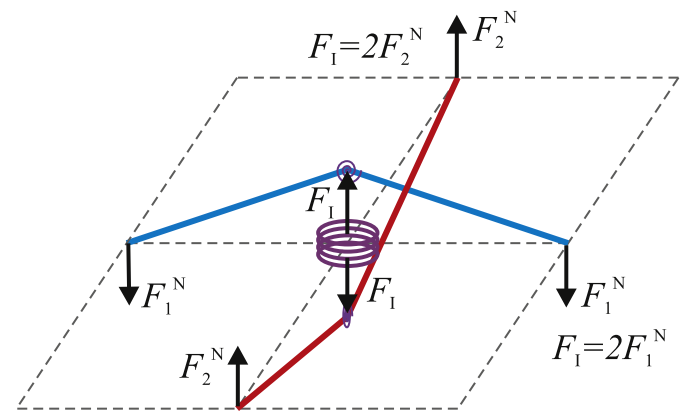

Fig. 5. Out-of-plane equilibrium of the fabric: the net through-thickness force in each yarn truss balances the force in the cross-over spring. 


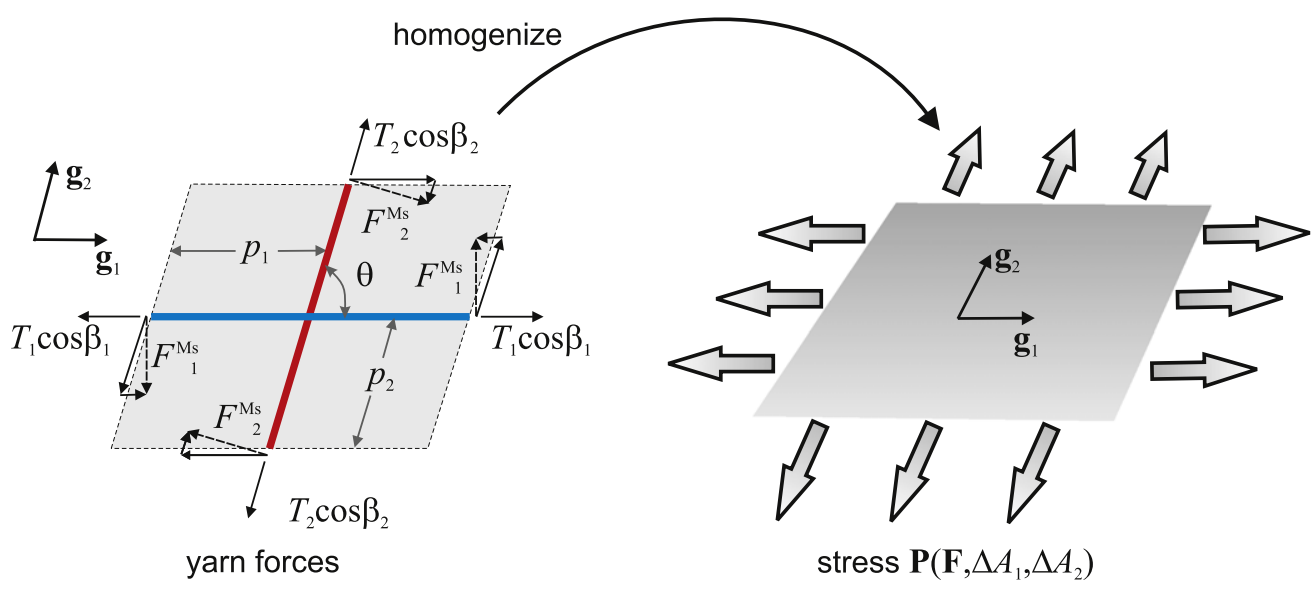

Fig. 6. Homogenization scheme: the in-plane forces on the yarns are projected onto the unit cell boundaries and converted into a continuum-level stresses. (Bending and locking forces omitted for clarity.)

the boundary of the unit cell and homogenized over the surface, resulting in stress tensor,

$$
\begin{aligned}
\mathbf{P}= & \frac{1}{2 p_{2}^{0} t_{\mathrm{f}}^{0}}\left\{T_{1} \cos \beta_{1}-\frac{M_{\mathrm{s}} \cos \theta}{2 p_{1} \sin \theta}\right\}\left(\mathbf{g}_{1} \otimes \mathbf{g}_{1}^{0}\right)+\frac{1}{2 p_{1}^{0} t^{0}}\left\{T_{2} \cos \beta_{2}-\frac{M_{\mathrm{s}} \cos \theta}{2 p_{2} \sin \theta}\right\}\left(\mathbf{g}_{2} \otimes \mathbf{g}_{2}^{0}\right) \\
& +\frac{1}{2 p_{1}^{0} t_{\mathrm{f}}^{0}}\left\{\frac{M_{\mathrm{s}}}{2 p_{2} \sin \theta}\right\}\left(\mathbf{g}_{1} \otimes \mathbf{g}_{2}^{0}\right)+\frac{1}{2 p_{2}^{0} t^{0}}\left\{\frac{M_{\mathrm{s}}}{2 p_{1} \sin \theta}\right\}\left(\mathbf{g}_{2} \otimes \mathbf{g}_{1}^{0}\right),
\end{aligned}
$$

where $t_{\mathrm{f}}^{0}$ is the initial thickness of the fabric. The forces due to the bending springs and locking trusses produce analogous terms but have been omitted here for the purpose of clarity.

\subsection{Woven fabric finite elements}

Triangular and quadrilateral finite elements have been developed and implemented into ABAQUS/Explicit via the VUEL user subroutine. The degrees of freedom, $\mathbf{d}$, are the three components of macroscopic displacement, $u_{i}$, and the changes in crimp amplitude of the yarns, $\Delta A_{1}$ and $\Delta A_{2}$. These degrees of freedom are explicitly integrated forward in time by the Newmark method. At $t^{n+1}$,

$$
\begin{aligned}
& \mathbf{d}_{n+1}=\mathbf{d}_{n}+\Delta t \dot{\mathbf{d}}_{n}+\frac{\Delta t^{2}}{2} \ddot{\mathbf{d}}_{n}, \\
& \ddot{\mathbf{d}}_{n+1}=\mathbf{M}^{-1}\left(\mathbf{f}_{n+1}^{\mathrm{ext}}+\mathbf{f}_{n+1}^{\mathrm{int}}\right),
\end{aligned}
$$

and

$$
\dot{\mathbf{d}}_{n+1}=\dot{\mathbf{d}}_{n}+\frac{\Delta t^{2}}{2}\left[\left(\ddot{\mathbf{d}}_{n}+\ddot{\mathbf{d}}_{n+1}\right)\right] .
$$

$\mathbf{M}$ is the diagonal mass matrix and $\mathbf{f}^{\text {ext }}$ and $\mathbf{f}^{\text {int }}$ are, respectively, the external and the internal nodal force vectors.

Fully integrated six-node triangular and nine-node quadrilateral membrane elements were used to simulate the fabric. An additional full set of "dummy" nodes was created for each element. The $u_{1}$ and $u_{2}$ "displacement" degrees of freedom of each dummy node represent the changes in crimp amplitude of the yarns.

A membrane formulation was chosen because out-of-plane bending and twist provide very small contributions to the ballistic impact resistance of woven fabrics. The overwhelming majority of the resistance to ballistic impact is provided by the in-plane deformation of the fabric. (Out-of-plane inertia is also important for large or multi-layer fabrics.) The out-ofplane resistance can cause the fabric to wrinkle (Hamila et al., 2009) but typically only at shear angles larger than those observed in these simulations. In order to verify that the membrane approximation was sufficiently accurate, we simulated a quasi-static indentation test of length scale similar to that of the ballistic impact experiments (Appendix A) with both a membrane element implementation of the constitutive model of King et al. (2005) and the analogous shell formulation of King and Socrate (2005). The constitutive response of the shell elements included resistances to out-of-plane bending and twist, both measured experimentally. The simulation with shell elements exhibited a force-displacement response virtually identical to the simulation with membrane elements (Fig. 30). We therefore determined that the additional complexity and cost of shell elements were not necessary.

The deformation gradient at each material point is calculated from the nodal positions and shape functions of the isoparametric elements. The in-plane position of a material point in the reference configuration is given, as a function of the natural coordinates, $\xi_{s}$ ( $s=1,3$ for triangular elements; $s=1,2$ for quadrilateral elements) by the shape functions at each 
node, $a$ :

$$
X_{J}\left(\xi_{s}\right)=N_{a}\left(\xi_{s}\right) X_{J a} \quad(J=1,2) .
$$

Correspondingly, the three-dimensional position of a material point in the current configuration is given by

$$
x_{i}\left(\xi_{s}\right)=N_{a}\left(\xi_{s}\right) x_{i a} \quad(i=1,3) .
$$

The relevant components of the deformation gradient at a point within the element are therefore calculated as

$$
F_{i J}=\frac{\partial x_{i}}{\partial X_{J}}=N_{a J} x_{i a} .
$$

Provided that the fabric initially lies in the 1-2 plane, $\mathbf{F}$ determines the orientation and stretch of the yarn vectors, $\mathbf{g}_{1}$ and $\mathbf{g}_{2}$, at each integration point within the element.

The internal nodal forces are calculated by the principle of virtual work. The internal nodal force corresponding to each displacement degree of freedom is calculated by integrating numerically over each element $e$ the standard expression,

$$
\left(f_{i a}^{\text {int }}\right)^{\mathrm{e}}=-\int_{\Omega_{0}^{\mathrm{e}}} P_{i j}\left(\mathbf{F}, \Delta A_{1}, \Delta A_{2}\right) N_{a J} \mathrm{~d} V_{0},
$$

and summing the contributions of each element to node $a$. In order to compute the internal nodal force corresponding to each crimp amplitude degree of freedom, we multiply Eq. (11) by virtual changes in crimp amplitude $\tilde{\Delta A_{k}}$ and integrate over the element. The result is the statement of transverse equilibrium in weak form (excluding inertial and external forces):

$$
\int_{\Omega_{0}^{e}} \tilde{\Delta A_{k}} \frac{F_{\mathrm{I}}\left(\Delta A_{1}, \Delta A_{2}\right)-2 F_{k}^{\mathrm{N}}\left(\mathbf{F}, \Delta A_{1}, \Delta A_{2}\right)}{4 p_{1}^{0} p_{2}^{0}} \mathrm{~d} S_{0}=0 \quad(k=1,2) .
$$

With $\tilde{\Delta A_{k}}\left(\xi_{s}\right)=N_{a}\left(\xi_{s}\right) \tilde{\Delta A_{k a}}$, the contribution to the internal nodal force vector from each element becomes

$$
\left(f_{(k+3) a}^{\text {int }}\right)^{\mathrm{e}}=\int_{\Omega_{0}^{\mathrm{e}}} \frac{F_{\mathrm{l}}\left(\Delta A_{1}, \Delta A_{2}\right)-2 F_{k}^{\mathrm{N}}\left(\mathbf{F}, \Delta A_{1}, \Delta A_{2}\right)}{4 p_{1}^{0} p_{2}^{0}} N_{a} \mathrm{~d} S_{0},
$$

which is also integrated numerically.

The mass of the fabric is apportioned approximately equally to each node in the finite element mesh. The total mass of each element is

$$
M=\rho_{\mathrm{f}}^{0} t_{\mathrm{f}}^{0} \frac{1}{2} W^{q} \sum_{q=1} J^{q},
$$

where $\rho_{\mathrm{f}}^{0}$ and $t_{\mathrm{f}}^{0}$ are the initial density and thickness, respectively, of the fabric and $J^{q}$ and $W^{q}$ are the Jacobian determinant and weight, respectively, at integration point $q$. Within each element, corner nodes, shared by more elements on average than mid-side nodes, receive proportionally less of the mass of the element than mid-side nodes. In order to calculate the mass associated each crimp amplitude degree of freedom, we imagine a unit cell of mass $M$ and take $L_{1} \approx L_{2} \equiv L$, $\beta_{1} \approx \beta_{2} \equiv \beta \approx 0$, and $m_{1}=m_{2} \equiv m=M / 4$. The inertia force resisting the rotation of a single half-length of yarn about its point of intersection with the boundary of the unit cell is

$$
J \ddot{\beta}=\frac{m L^{2}}{3} \ddot{\beta}=\frac{M L^{2}}{12} \ddot{\beta} \approx \frac{1}{2}\left(F_{1}-2 F_{k}^{N}\right) L,
$$

where $J$ is the moment of inertia of the half-length of yarn (Fig. 7). With the out-of-plane acceleration at the cross-over point approximated as $\ddot{A}_{k} \approx \ddot{\beta} L$, the transverse equilibrium equations become

$$
\frac{M}{6} \Delta A_{k}=F_{\mathrm{I}}-2 F_{k}^{\mathrm{N}} \quad(k=1,2) .
$$

Thus, at each node, the mass associated with the crimp amplitude degrees of freedom is one-sixth of the mass associated with the displacement degrees of freedom.

Element failure occurs when the element-average tension in either yarn family exceeds the strength of the yarn, $T_{k}>T_{k}^{\max }$. This failure criterion assumes that, once one yarn family has broken in an element, the projectile will easily push the other yarn family aside. When an element fails, its contribution to the internal nodal force vector, $\mathbf{f}^{\text {int }}$, is set to zero.

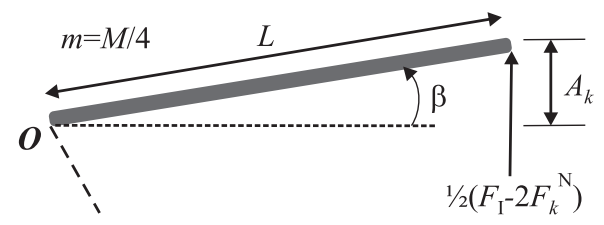

Fig. 7. Calculation of lumped mass corresponding to amplitude degrees of freedom. 


\subsection{Quasi-static verification of fabric model}

The accuracy at low strain rates of the fabric finite elements was verified by simulating the quasi-static tests of King et al. King et al. loaded strips of Kevlar S706 in uniaxial tension at a nominal axial strain rate of $0.01 \mathrm{~s}^{-1}$. Three cases were considered: (1) warp yarns parallel to the loading direction ("warp"); (2) weft yarns parallel to the loading direction ("weft"); (3) both yarn families oriented at $45^{\circ}$ to the loading direction ("bias extension"). The warp and weft strips measured $2.54 \mathrm{~cm} \times 25.4 \mathrm{~cm}$ and were simulated with 500 six-node triangular membrane elements. The bias extension strips measured $3.5 \mathrm{~cm} \times 9.5 \mathrm{~cm}$ and were simulated with 298 six-node triangular membrane elements. In all three cases, the experiment and the simulation agree (Figs. 8 and 9). The initial, nearly flat portions of the warp and weft results are due to crimp interchange: the yarns parallel to the loading direction extend simply by uncrimping, not stretching. At strains of $1-2 \%$, the yarns straighten out, and they then begin to stretch and offer significant resistance to deformation. The weft strips stiffen at larger strains than the warp strips because the initial crimp amplitude of the weft yarns is $50 \%$ greater than the initial crimp amplitude of the warp yarns $(0.090 \mathrm{~mm}$ versus $0.060 \mathrm{~mm}$, respectively). The bias strips exhibit a much more compliant response than the warp and weft strips. They deform with little resistance until strains of about $20 \%$. The deformation of the bias strips is accommodated primarily by easy rotation of the yarns toward the axis of loading. At large strains, however, as the yarns jam against one another, the resistance to rotation increases, and stretching of the yarns plays an increasingly important role. It is this large deformation at small loads enabled by yarn rotation that makes woven fabric an ideal reinforcement phase for complex composite parts. During forming processes, fabrics are able to conform to molds with compound curvature without the yarns breaking.
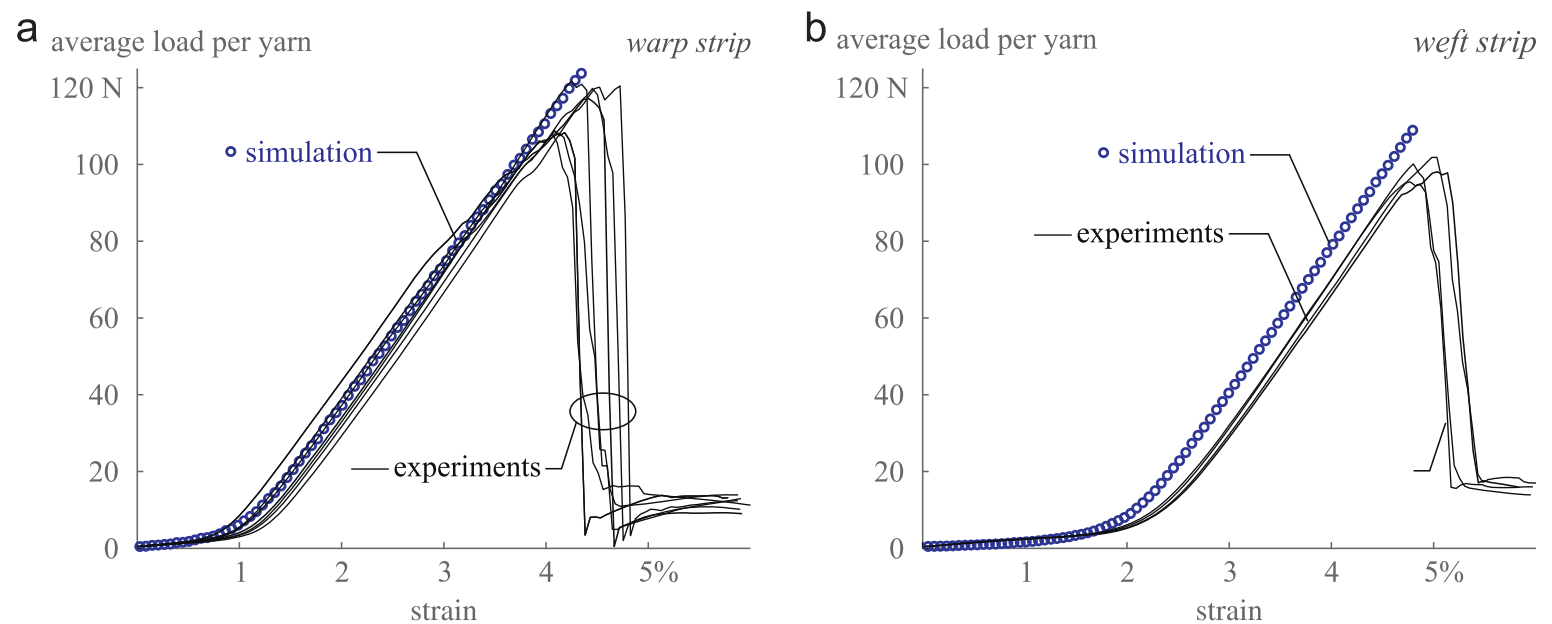

Fig. 8. Simulation versus experiments (King et al., 2005) for strips of Kevlar S706 under uniaxial tension in (a) warp direction and (b) weft direction.

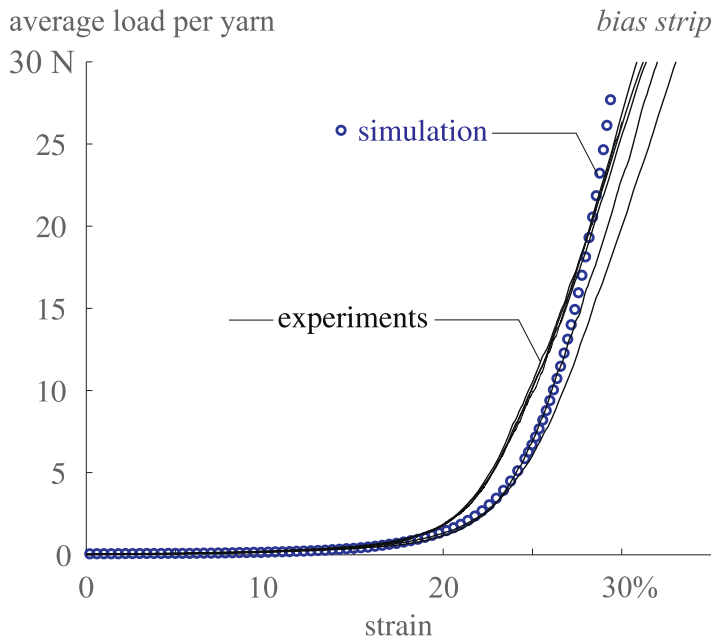

Fig. 9. Simulation versus experiments (King et al., 2005) for Kevlar S706 bias extension test. 


\section{Low velocity projectile impact experiments and simulations}

Woven fabrics such as Kevlar ${ }^{\circledR}$ S706 are frequently used for protection against various types of projectiles. During a successful projectile impact event, the fabric absorbs and disperses all of the kinetic energy of the projectile without deflecting excessively. The kinetic energy of the projectile is transformed predominantly into kinetic energy and strain energy of the yarns.

In this section, we demonstrate the accuracy of the fabric finite elements at elevated strain rates by rigorously comparing their predictions with the results of projectile impact experiments. Projectile impacts load fabrics at high rates, but the constitutive relations and material parameters of the woven fabric model were determined from tests at quasi-static rates. Cheng et al. (2005) showed that the uniaxial extension of KM2 yarns is nearly rate-independent, and we have assumed, with the exception of dissipative yarn rotation, that the other modes of deformation of the yarns are approximately rateindependent as well. In order to test this assumption, experiments with initial projectile velocities $v_{\mathrm{i}}=22-30 \mathrm{~m} / \mathrm{s}$ were performed on Kevlar S706 at the US Army Research Laboratory (ARL). The maximum strain rate in these experiments was about $25 \mathrm{~s}^{-1}$-not quite in the ballistic range, but several orders of magnitude greater than the strain rates in the quasistatic tests. These experiments were unique because, via three-dimensional digital image correlation, they provided the full displacement field of the back surface of the fabric (opposite the side of impact). The displacement fields of the experiments and the simulations are close matches, validating the rate assumptions of the model.

\subsection{Procedure for low velocity projectile impact (ARL) experiments}

Projectile impact experiments on single plies of Kevlar S706 were conducted at ARL with a gas gun. Approximately $40 \mathrm{~cm}$ square fabric specimens were cut from a roll of the fabric. Each specimen was stapled to a $30.5 \mathrm{~cm}$ square pictureframe with a pneumatic staple gun (Fig. 10). The warp yarns of the fabric were aligned horizontally (1-direction), and the weft yarns of the fabric were aligned vertically (2-direction). Frames with a $25.4 \mathrm{~cm}$ square cut-out and a $25.4 \mathrm{~cm}$ diameter circular cut-out were used. The stapled specimen was first spray painted white and then speckled with black paint. Next, the fabric and wooden frame were clamped between two square aluminum frames of dimensions similar to those of the wooden frame. The surfaces of the aluminum frames in contact with the fabric were roughened in order to limit slip of the fabric from the frame. One of the aluminum frames was fixed to a table at the outlet of the barrel of the gas gun such that the projectile struck the center of the fabric at a perpendicular angle. The gas gun was charged with nitrogen and fired the projectiles at initial velocities $v_{\mathrm{i}}=22-30 \mathrm{~m} / \mathrm{s}$. The steel, hemispherically nosed projectiles measured $12.7 \mathrm{~mm}$ in diameter and weighed $104 \mathrm{~g}$.

The initial velocity of the projectile and the full-field displacements were measured in the experiments (Fig. 11). The initial velocity of the projectile was measured at the outlet of the gun barrel with a three-laser beam detector system. This detector also triggered the two Photron APX-RS high speed digital cameras to start recording images. Images of the back surface of the specimen were acquired at a frequency of $30 \mathrm{kHz}$. Commercial digital image correlation (DIC) software from Correlated Solutions, Inc. was then used to compute the full-field in-plane $\left(u_{1}, u_{2}\right)$ and transverse $\left(u_{3}\right)$ displacements of the fabric.

While great care was taken in the experiments to minimize slip of the fabric from the frame, as with nearly all experiments of this type, some slip did occur. Cunniff (1992), Walsh et al. (1998), Shim et al. (1995), and Zeng et al. (2005), among others, also observed slip of the fabric in experiments. The fabric slipped a maximum of $2-5 \mathrm{~mm}$ of from each side of the frame, during both the square and circular cut-out tests. The location of maximum slip from each side was horizontally or vertically in line with the point of impact. For example, the DIC results for vertical displacement in Fig. 12 show in excess of $4 \mathrm{~mm}$ of slip from the top of the frame at the time of peak transverse displacement.

a

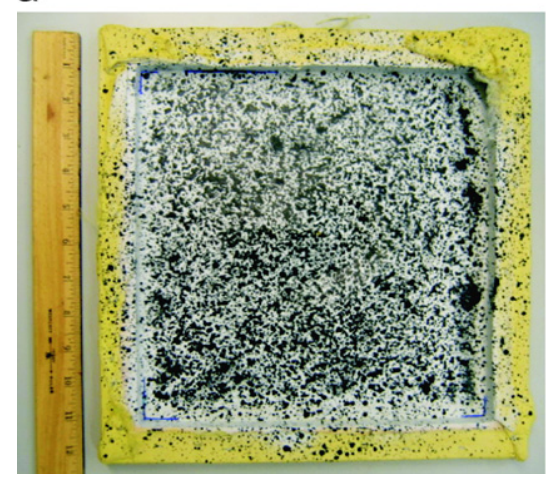

b

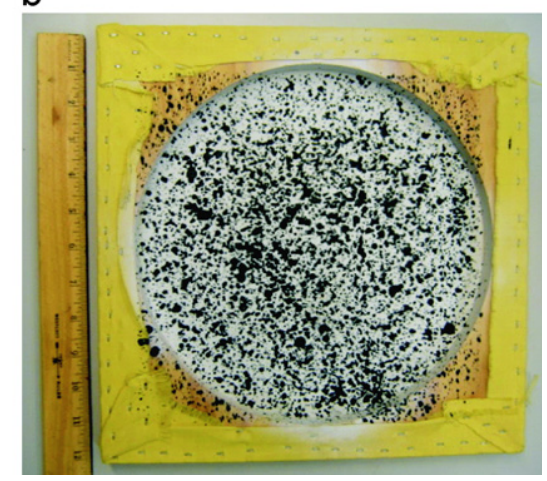

Fig. 10. Back view of Kevlar fabric stapled to wooden frame with (a) $25.4 \mathrm{~cm}$ square cut-out and (b) $R=12.7 \mathrm{~cm}$ circular cut-out. 


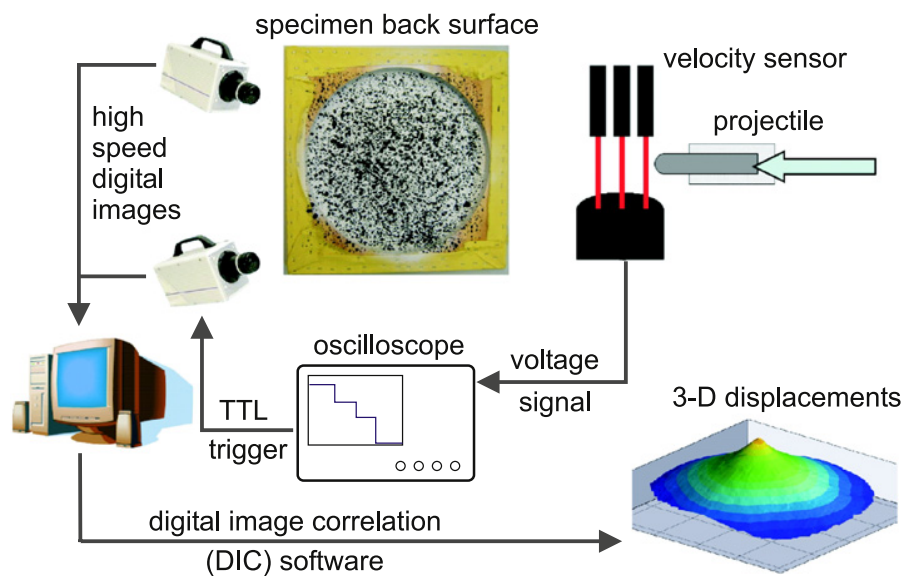

Fig. 11. Schematic of the ARL experimental set-up for measuring initial projectile velocity and full-field displacements during a projectile impact test.

a

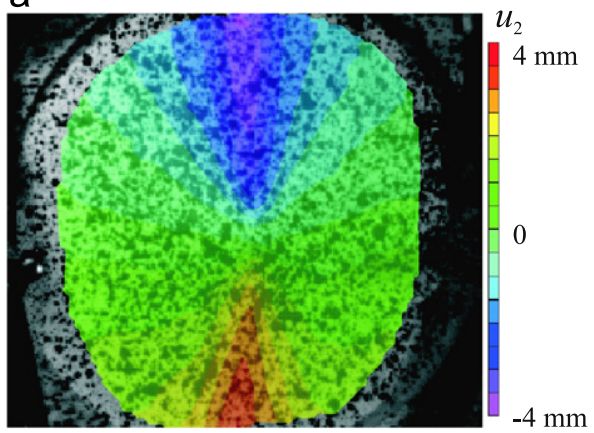

b

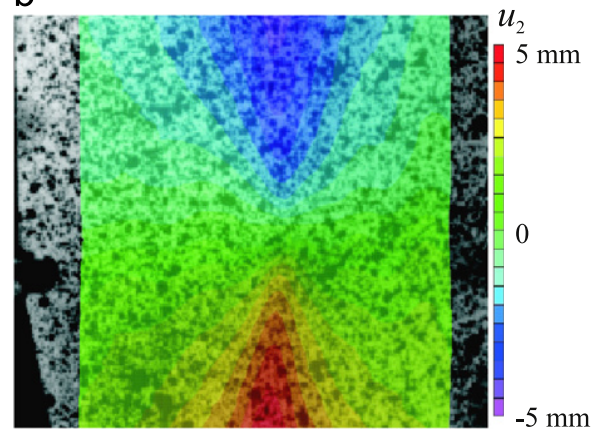

Fig. 12. Experimental slip of the fabric from top and bottom of the frame: contours of $u_{2}$-displacement for (a) circular cut-out at $t=2.0 \mathrm{~ms}$ and (b) square cut-out at $t=2.5 \mathrm{~ms}$.

\subsection{Procedure for simulations of low velocity projectile impact}

One-quarter of each fabric specimen was simulated with quadratic membrane elements. Approximately 3400 six-node, triangular elements were used, resulting in an average element edge length of about $5 \mathrm{~mm}$. The projectile was modeled with an analytical rigid surface. The sections of the fabric outside of the cut-outs were clamped with pressures of $600 \mathrm{kPa}$ (circular case) and $400 \mathrm{kPa}$ (rectangular case) between two rigid surfaces representing the wooden and aluminum frames. The clamping pressure in the simulations was chosen such that the average maximum slip of the fabric from the frame matched that measured during the experiment (Fig. 13 and Appendix B). The interactions between the projectile and the fabric, the aluminum frame and the fabric, and the wooden frame and the fabric were modeled with the kinematic predictor/corrector (hard) contact algorithm and the Coulomb friction model. Coefficients of friction for steel and aluminum sliding on Kevlar and other para-aramid fabrics have been shown to be in the range $\mu=0.20-0.31$ (Martínez et al., 1993; Lim et al., 2003; Tan et al., 2003). The coefficient of friction for all interactions was therefore approximated as $\mu=0.3$. The macroscopic response of the fabric depends very little on the fabric/projectile coefficient of friction, but it depends strongly on the clamping pressure and coefficient of friction between the fabric and the clamping surfaces. We show in Appendix B the effect of varying the clamping pressure on the amount of slip from the frame and the transverse deformation of the fabric. The failure criterion was $T_{k}^{\max }=112 \mathrm{~N}$, the average load per yarn at which the warp and weft strips failed in uniaxial tension (Fig. 8). The time of computation on a PC with $3 \mathrm{~GB}$ of RAM and a single Xeon ${ }^{\circledR} 3.2 \mathrm{GHz}$ CPU (4 MB L2 cache, $1066 \mathrm{MHz}$ front-side bus speed) was about $1 \mathrm{~h}$.

During the experiments, the fabric deformed almost identically in the warp and weft directions, indicating that the specimen was subject to a preload. Without a preload, the smaller initial crimp amplitude of the warp yarns $(0.060 \mathrm{~mm}$ versus $0.090 \mathrm{~mm}$ for the weft yarns) causes the transverse displacement waves to travel faster in the warp direction than in the weft direction. Preload of the fabric reduced and equalized the crimp of the yarns, eliminating the majority of the anisotropy between the two yarn directions. Practically speaking, it is next to impossible to clamp a fabric completely flat 

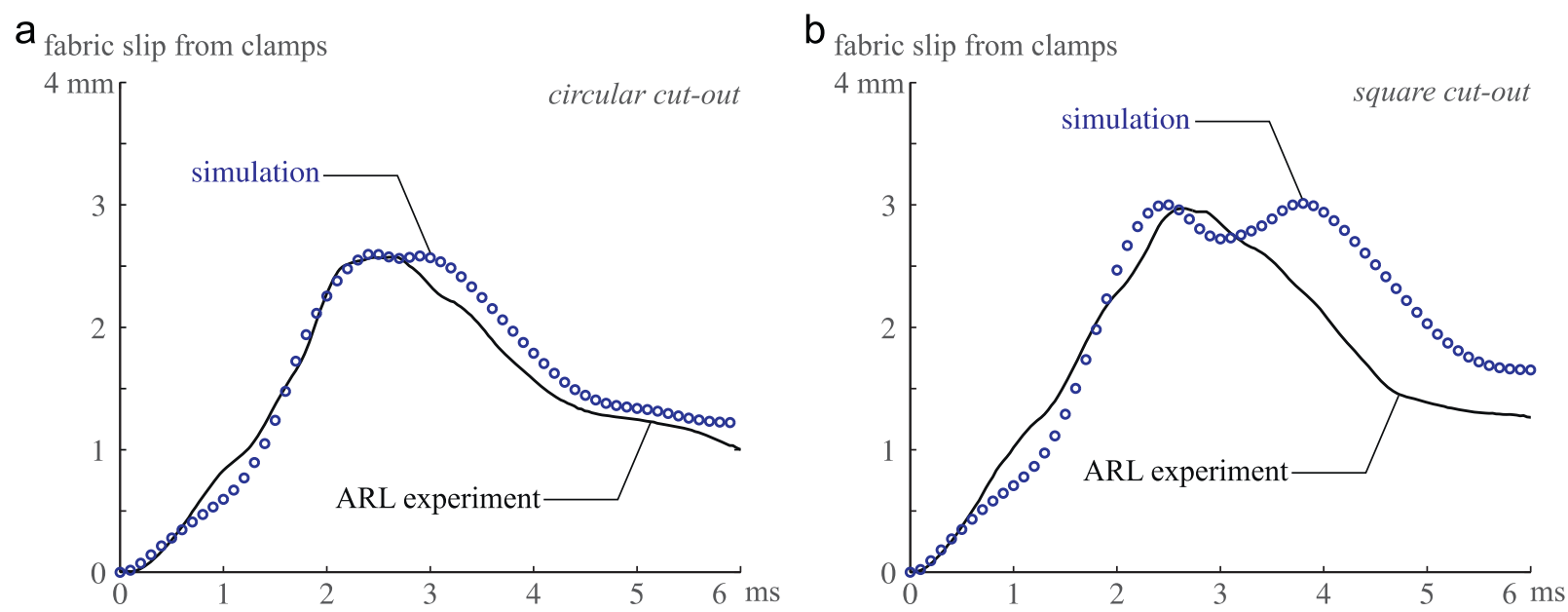

Fig. 13. Prediction of average in-plane slip displacement of the fabric from the frame with $v_{\mathrm{i}}=22 \mathrm{~m} / \mathrm{s}$ : (a) circular cut-out, (b) square cut-out.

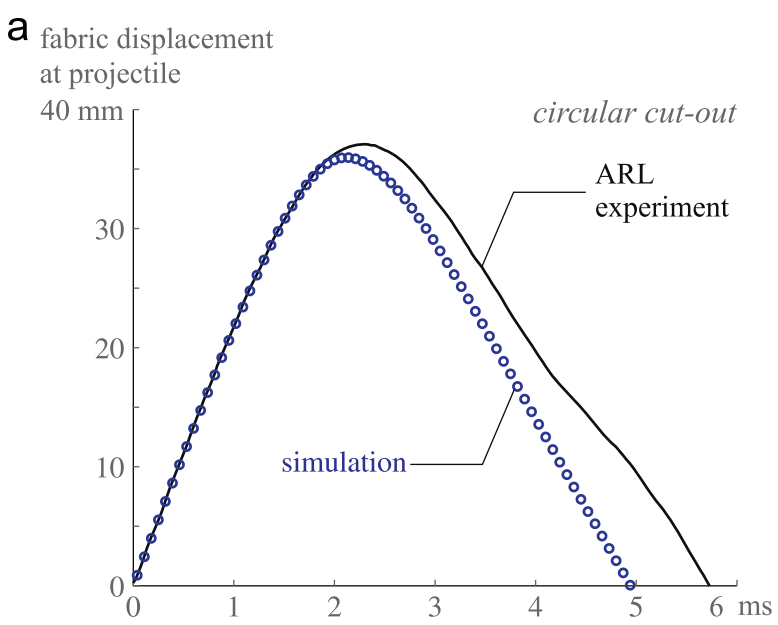

b fabric displacement
at projectile

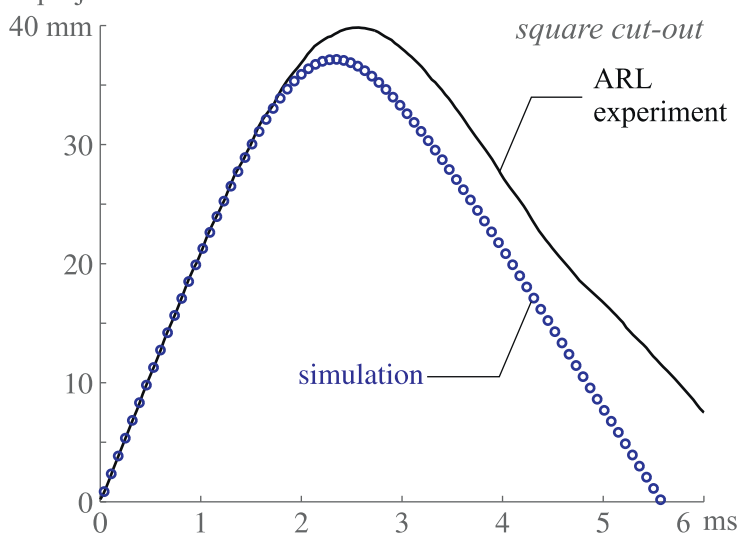

Fig. 14. Prediction of transverse displacement at point of impact for fabric with $v_{\mathrm{i}}=22 \mathrm{~m} / \mathrm{s}$ : (a) circular cut-out, (b) square cut-out.

without tensioning it. The preload was determined to be approximately $1 \mathrm{~N}$ per yarn. In the simulations, the preload was applied by specifying an initial deformation gradient, $\mathbf{F}^{\text {pre }}$, and initial changes in yarn crimp amplitude, $\Delta A_{1}^{0}$ and $\Delta A_{2}^{0}$. Further details of the preload procedure are provided in Appendix C.1.

\subsection{Results of low velocity projectile impact experiments and simulations}

At initial projectile velocity $v_{\mathrm{i}}=22 \mathrm{~m} / \mathrm{s}$, the fabric defeated the projectile in both experimental cases, and the fabric finite elements accurately simulate the deformation of the fabric by several standards of measurement. For the experiment with the circular cut-out, the history of transverse displacement of the fabric at the point of impact shows that the maximum displacement was $37.1 \mathrm{~mm}$ at $2.3 \mathrm{~ms}$ after impact (Fig. 14a). In the experiment with the square cut-out, the maximum displacement was $39.8 \mathrm{~mm}$ at $2.6 \mathrm{~ms}$ after impact (Fig. 14b). The simulations underpredict the maximum displacement of the fabric by $1 \mathrm{~mm}$ in the circular case and $2.6 \mathrm{~mm}$ in the square case, but overall they compare very well with the experiments, even during the rebound phase of the deformation. The experimental full-field displacements, computed by digital image correlation, provide a more comprehensive test of the fabric finite elements. Figs. 15 and 16 show that the deformed shapes and full-field contours of transverse displacement of the experiments and simulations look very similar. The deformed shape of the fabric in both the experiments and the simulations resembles a pyramid due to the anisotropy of the fabric. When the projectile contacts the fabric, transverse displacement waves travel along the impacted or "primary" yarns away from the point of impact. Initially, the primary yarns form the inclined edges of the pyramid, and the base of the pyramid is diamond-shaped (Figs. 15a-b', 16a-b'). The transverse waves may only travel along the yarns, and 
ARL experiment:

a

$0.4 \mathrm{~ms}$ :

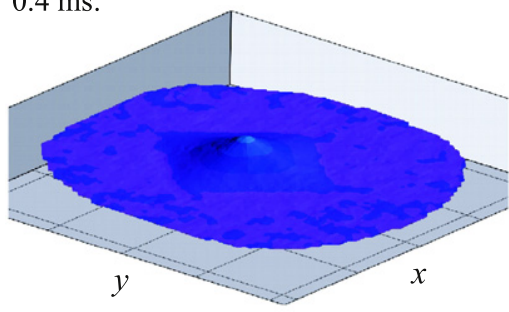

b

$0.9 \mathrm{~ms}$ :

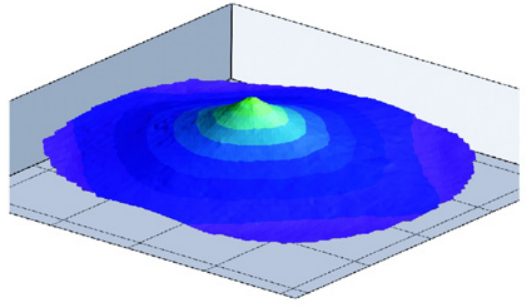

C

$1.3 \mathrm{~ms}$ :

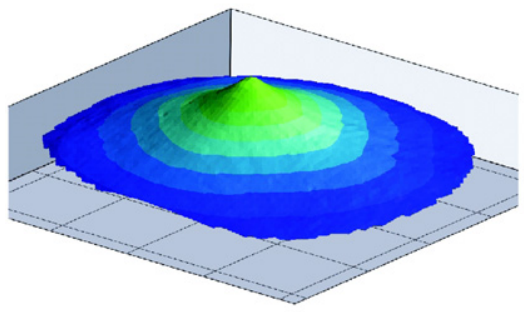

d

$2.0 \mathrm{~ms}$ :

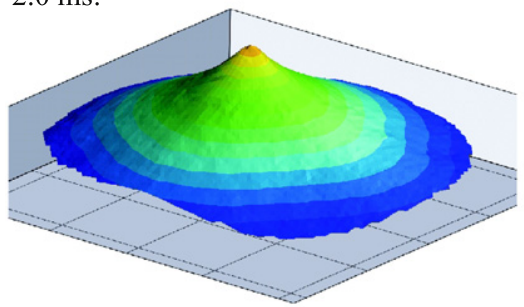

simulation:

a'
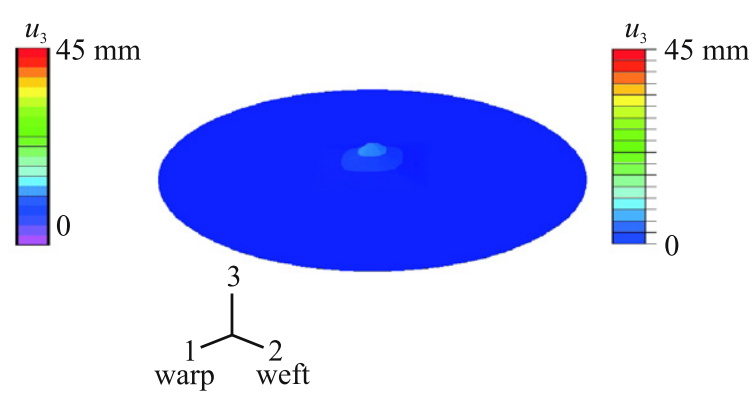

b'

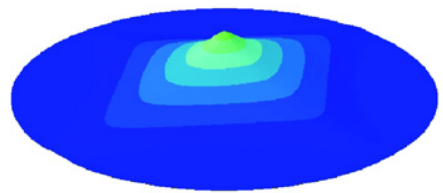

$C^{\prime}$

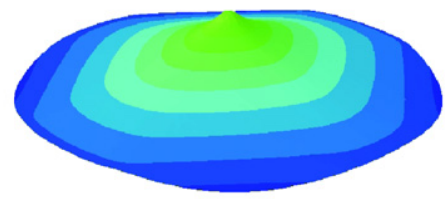

d'

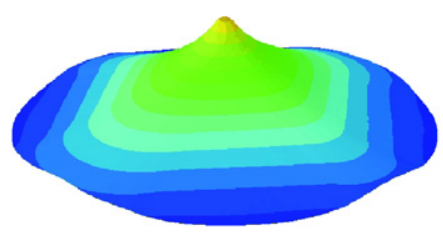

Fig. 15. Contours of transverse displacement for experiment (left) and simulation (right) for circular cut-out with $v_{\mathrm{i}}=22.2 \mathrm{~m} / \mathrm{s}$ at time after impact: (a), (a') $0.4 \mathrm{~ms}$; (b), (b') $0.9 \mathrm{~ms}$; (c), (c') $1.3 \mathrm{~ms}$; (d), (d') $2.0 \mathrm{~ms}$.

therefore the yarns not in contact with the projectile, the "secondary" yarns, do not begin to deflect until their crossing primary yarns deflect. When the transverse waves reach the frame, the base of the pyramid spreads, and the number of facets of the pyramid doubles (Figs. $15 c-d^{\prime}, 16 c-d^{\prime}$ ). The simulations predict the correct shape of the fabric because the elements capture the anisotropy of the system of discrete yarns. In contrast, isotropic continuum-level finite elements would predict the transverse displacement waves to travel radially in all directions, resulting in a cone shape to the fabric. Finally, for a more quantitative evaluation of the elements, we plot in Figs. 17 and 18, for both experiments and simulations, the transverse displacement profiles along lines in the warp and weft directions through the point of impact. At all three times, with the final time corresponding approximately to the maximum displacement of the fabric, the transverse displacements of the simulations and the experiments match closely. The warp and weft profiles are nearly identical. The preload of the fabric has reduced the initial crimp of the warp and weft yarns, the primary source of anisotropy between the warp and weft directions. Without the preload, the transverse displacement waves would travel more slowly, particularly in the weft direction, because of the additional uncrimping required. 
ARL experiment:

a

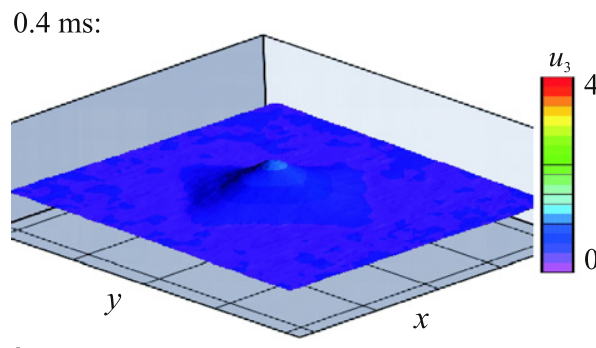

b

$0.9 \mathrm{~ms}$ :

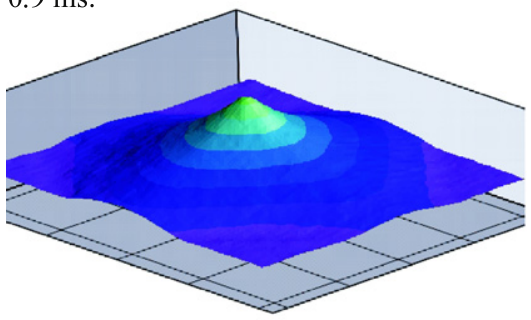

C

$1.3 \mathrm{~ms}$ :

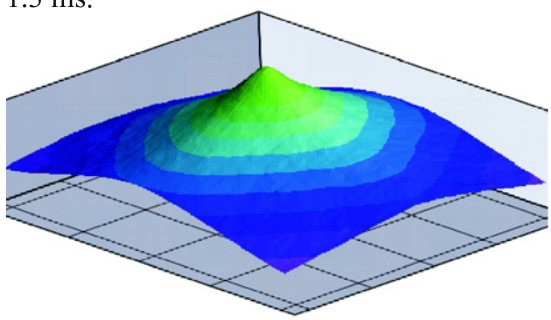

d

$2.5 \mathrm{~ms}$ :

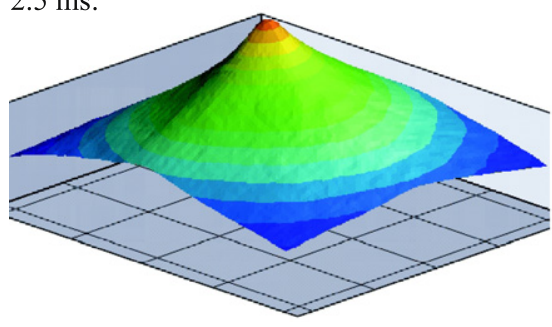

simulation:

a'
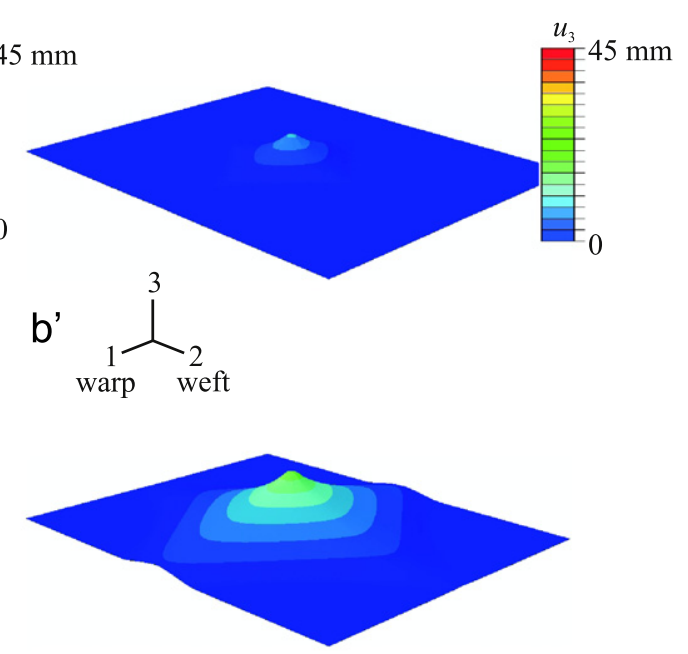

$c^{\prime}$

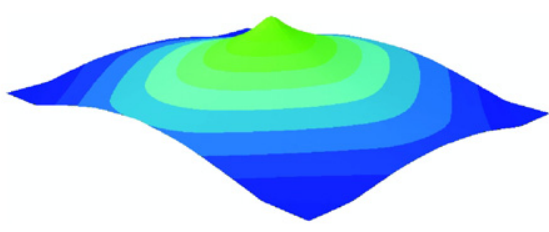

d'

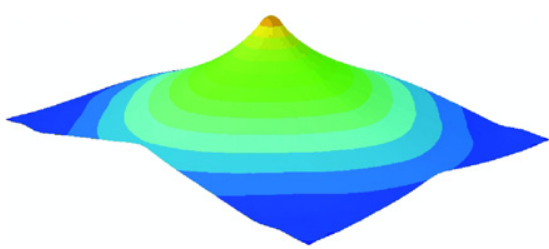

Fig. 16. Contours of transverse displacement for experiment (left) and simulation (right, in $\mathrm{m}$ ) for square cut-out with $v_{\mathrm{i}}=22 \mathrm{~m} / \mathrm{s}$ at time after impact: (a), (a') $0.4 \mathrm{~ms}$; (b), (b') $0.9 \mathrm{~ms}$; (c), (c') $1.3 \mathrm{~ms}$; (d), (d') $2.5 \mathrm{~ms}$.

The fabric finite elements are accurate at the macroscopic level because their predictions are built upon the actual deformation of the yarns. The deformation of the yarns is tracked in the elements by state variables characterizing the forces in the yarns and the geometry of the fabric. Fig. 19a-d displays, for the circular cut-out, a snapshot of several key yarn properties at $t=2.0 \mathrm{~ms}$, which is close to the time of maximum transverse displacement. A band of high tension has developed in the primary warp yarns (Fig. 19a). These are the warp yarns that carry the majority of the impact load. The maximum warp yarn tension, $T_{1} \approx 100 \mathrm{~N}$ at the point of impact, is dangerously close to strength of the yarns, $T_{k}^{\max }=112 \mathrm{~N}$. Although not shown, the weft yarns exhibit a similar band of high tension, causing the pattern of the highly loaded warp and weft yarns together to resemble a cross. The crimp amplitude of the primary warp yarns has decreased, while at $90^{\circ}$, where the weft yarns carry the majority of the load, the crimp amplitude of the warp yarns has increased (Fig. 19b). The stretch and uncrimping of the primary warp yarns has pulled the crossing weft yarns apart, causing the weft yarn spacing, $2 p_{1}$, to increase along the primary warp yarns (Fig. 19c). In the secondary yarns, particularly near the projectile, sizable yarn rotation angles, $\gamma$, have developed (Fig. 19d), indicating that the macroscopic deformation of the fabric is enabled not only by stretching of the yarns but also by significant shearing of the yarns. 

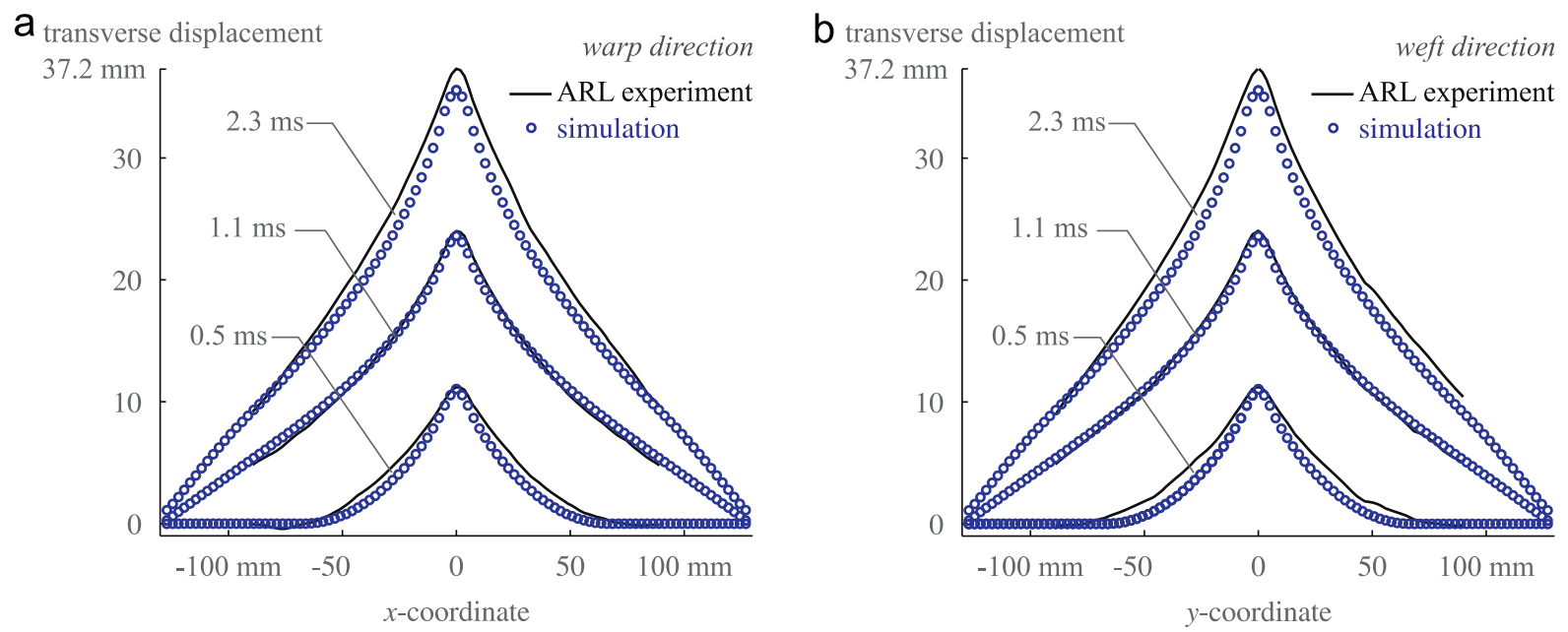

Fig. 17. Prediction of transverse displacement profiles through point of impact for circular cut-out with $v_{\mathrm{i}}=22 \mathrm{~m} / \mathrm{s}$ : (a) warp direction through point of impact, (b) weft direction through point of impact.
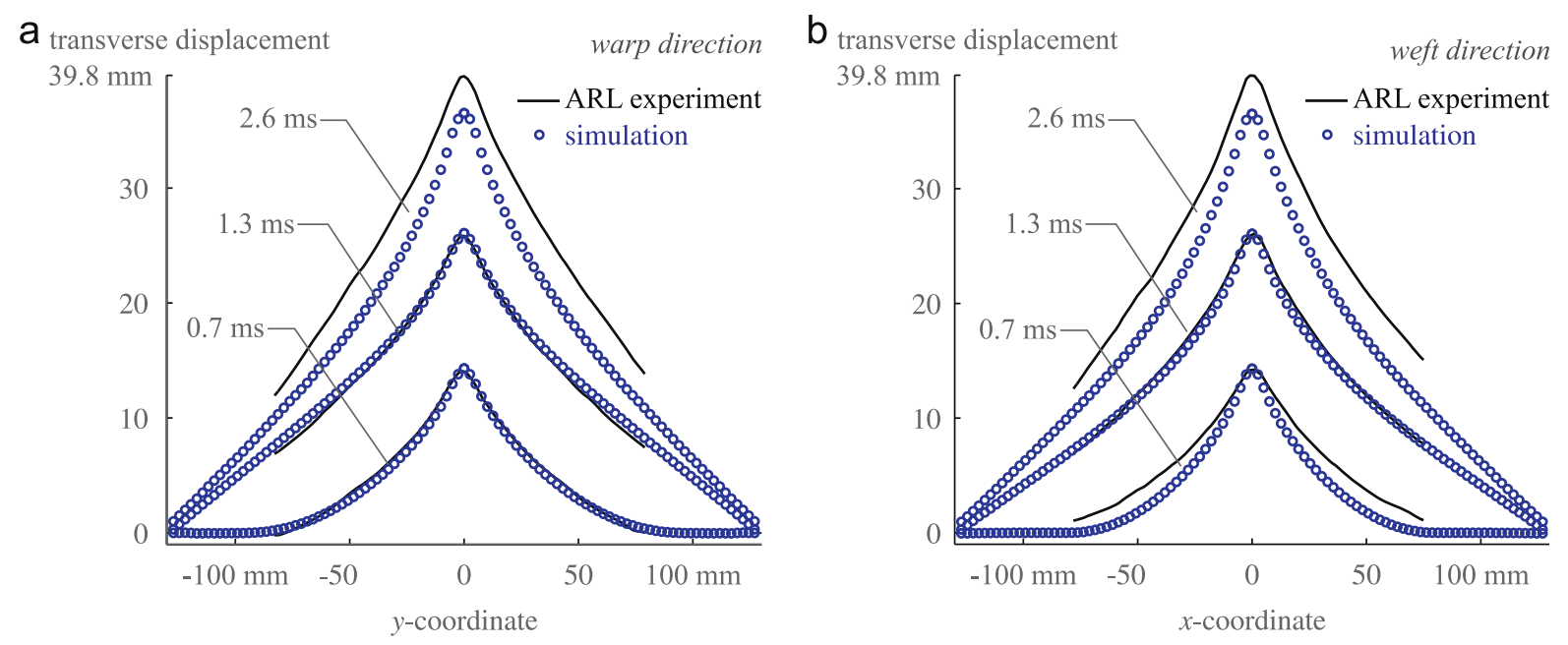

Fig. 18. Prediction of transverse displacement profiles through point of impact for square cut-out with $v_{\mathrm{i}}=22 \mathrm{~m} / \mathrm{s}$ : (a) warp direction through point of impact, (b) weft direction through point of impact.

The tracking of yarn tension permits the finite elements to predict the failure of the fabric. In the experiments, failure occurred when the initial velocity of the projectile was increased to $30 \mathrm{~m} / \mathrm{s}$. At this projectile velocity, the simulation predicts failure at the appropriate time, $t \approx 1.4 \mathrm{~ms}$, and the contours of transverse displacement of the experiment and simulation agree (Fig. 20). The simulation predicts the residual velocity of the projectile to be $15.4 \mathrm{~m} / \mathrm{s}$. The residual velocity was not measured in the experiment.

\section{Ballistic impact experiments and simulations}

Although complex, the mechanics of ballistic impact of yarns and woven fabrics is well characterized in the literature by Smith et al. (1956), Roylance et al. (1973), Roylance (1980), and Ting et al. (1998), among others. When a projectile strikes a woven fabric, longitudinal strain waves propagate along the primary yarns away from the point of impact. In the wake of these strain waves, the yarns translate inward toward the projectile. At the cross-over points, portions of the strain waves are diverted along the orthogonal secondary yarns. When a longitudinal strain wave reaches a fixed edge, it is reflected and the strain in the yarn behind the reflected wave front increases. A longitudinal strain wave is reflected by a free edge as well, but behind the wave front in this case, the strain disappears and the velocity of the yarn moving inward toward the projectile increases. At the time of impact, transverse displacement waves also develop in the primary yarns and propagate away from the projectile more slowly than the longitudinal waves. As discussed in Section 3, at cross-over points, portions of the transverse displacement waves are diverted along the orthogonal secondary yarns, causing the pyramidal shape of the fabric. The outward velocities of the transverse displacement waves are proportional to the strain in the yarns. The 

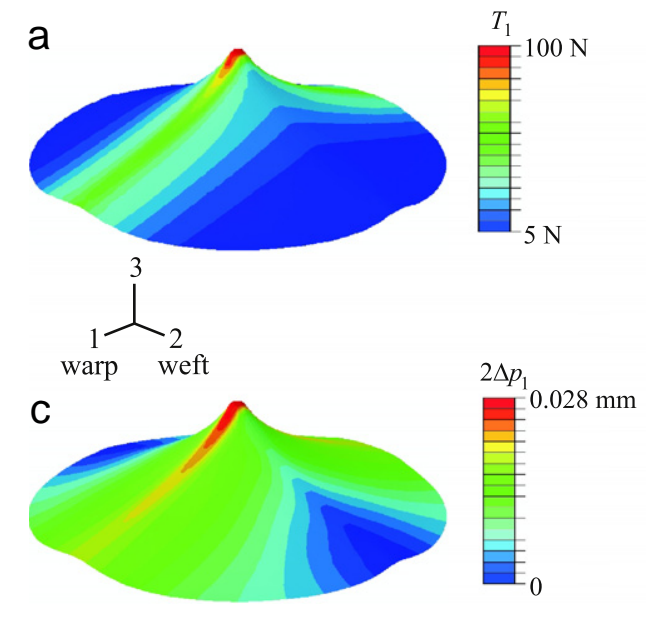
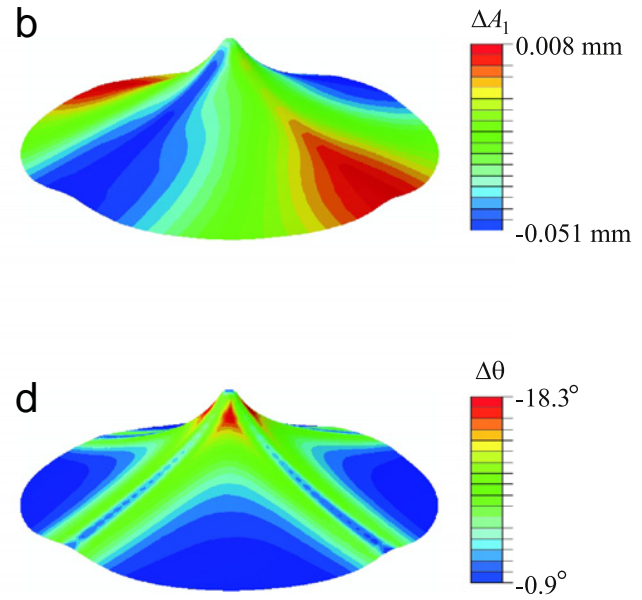

Fig. 19. Contours of yarn-level quantities during simulation of circular cut-out with $v_{\mathrm{i}}=22.2 \mathrm{~m} / \mathrm{s}$ at $t=2.0 \mathrm{~ms}$ : (a) warp yarn tension, (b) change in warp yarn crimp amplitude, (c) change in weft yarn spacing, (d) shear angle.

transverse displacement waves are reflected at the edges of the fabric, giving rise to new longitudinal strain waves. Both the longitudinal and the transverse waves are again reflected upon their return to the projectile, and so forth.

Ballistic impact experiments performed on Kevlar S706 were also successfully simulated with the fabric finite elements. In these experiments, the gage section of the fabric was square, and the fabric was clamped on only two edges. The initial velocity of the projectile was measured with a laser sensor. Images of the deforming specimen were recorded with a high speed CCD camera. The images were used to measure the transverse displacement of the fabric and the residual velocity of the projectile for comparison with the simulations. The deformations in the experiments and simulations were more complicated than those of the theoretical case because, due to slip of the fabric, the clamped edges were neither fixed nor free.

\subsection{Procedure for ballistic impact (ISN) experiments}

The ballistic impact tests were performed with a gas gun (Fig. 21a) at the MIT Institute for Soldier Nanotechnologies (ISN). This gas gun was a single stage type with a breech capacity of $69 \mathrm{MPa}$ and a barrel $12.7 \mathrm{~mm}$ in diameter and $2.13 \mathrm{~m}$ long. The breech comprised two chambers separated from the projectile by a pair of diaphragms. Upon pressurization of the breech, the diaphragms burst, and the projectile was propelled along the horizontal barrel. The cylindrical polycarbonate projectiles weighed $3.6 \mathrm{~g}$ and were $12.65 \mathrm{~mm}$ in diameter with an approximately hemispherical tip. The chamber pressures, diaphragm material (aluminum or Mylar ${ }^{\mathbb{R}}$ ), diaphragm thickness, and pressurization gas (nitrogen or helium) were varied in order to produce initial projectile velocities in the range $v_{\mathrm{i}}=150-550 \mathrm{~m} / \mathrm{s}$. The initial velocity of the projectile was measured with two parallel laser ribbons spaced $15.24 \mathrm{~cm}$ apart. The intensity of the laser ribbons was monitored with photodiodes, which indicated when the projectile interrupted the lasers. After exiting the barrel, the projectile entered a closed chamber containing at its end the fabric specimen. The fabric was aligned perpendicularly to the barrel of the gun, which was sited at the center of the fabric. Ports in the chamber wall provided views parallel and at $45^{\circ}$ to the fabric.

The fabric specimens were rectangular single plies of Kevlar S706 with a $15.2 \mathrm{~cm}$ square gage section. The specimens measured $15.2 \mathrm{~cm} \times 38.1 \mathrm{~cm}$, with the warp yarns aligned with the long edges. Starting from the short edges, approximately $11.4 \mathrm{~cm}$ of each end of the fabric was wrapped around a steel bar of $9.5 \mathrm{~mm}$ square cross-section (Fig. 21b). Each fabric wrapped bar was then fixed between a set of steel clamps such that the warp yarns were aligned vertically (Fig. 21c). One clamp from each set was bolted to a frame mounted at the end of the chamber, and the mating clamps were in turn bolted to the clamps on the frame. The clamp separation was $15.24 \mathrm{~cm}$. The clamps were knurled in order to minimize slip of the fabric.

A Cordin CCD camera with a rotating mirror photographed the experiments through one of the ports in the chamber wall. Equipped with a Nikon $70-300 \mathrm{~mm}$ lens, the camera was triggered by the initial velocity sensor at a delay. The camera triggered a high performance strobe light and recorded 1 megapixel images at 32 equal intervals. The length of the delay and the interval between images was determined from the estimated initial velocity of the projectile. The displacement of the fabric and the residual velocity of the projectile were measured manually from the images. The system did have a residual velocity sensor, but it was not possible to use the sensor and record images of the experiment simultaneously. (The residual velocity sensor blocked the view through the port.)

\subsection{Procedure for simulations of ballistic impact}

One-quarter of each fabric specimen was simulated with quadratic membrane elements. The finite element mesh consisted of 555 nine-node quadrilateral elements for an element edge length of about 5 mm. The projectile was modeled 
ARL experiment:

a

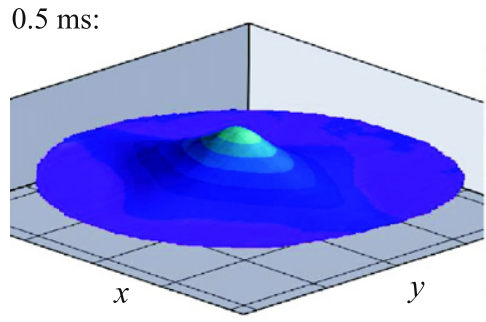

b

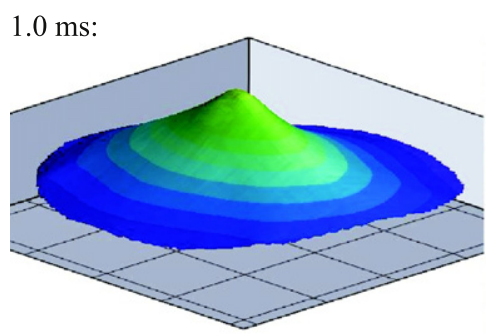

C

$1.425 \mathrm{~ms}$ :

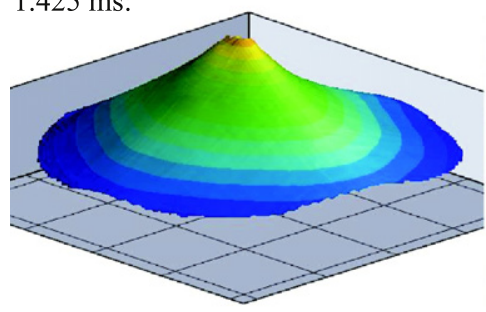

d

$1.575 \mathrm{~ms}$ :

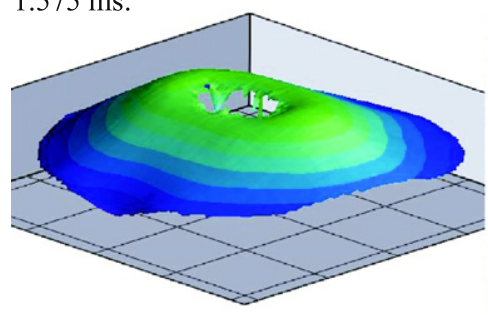

simulation:

$a^{\prime}$
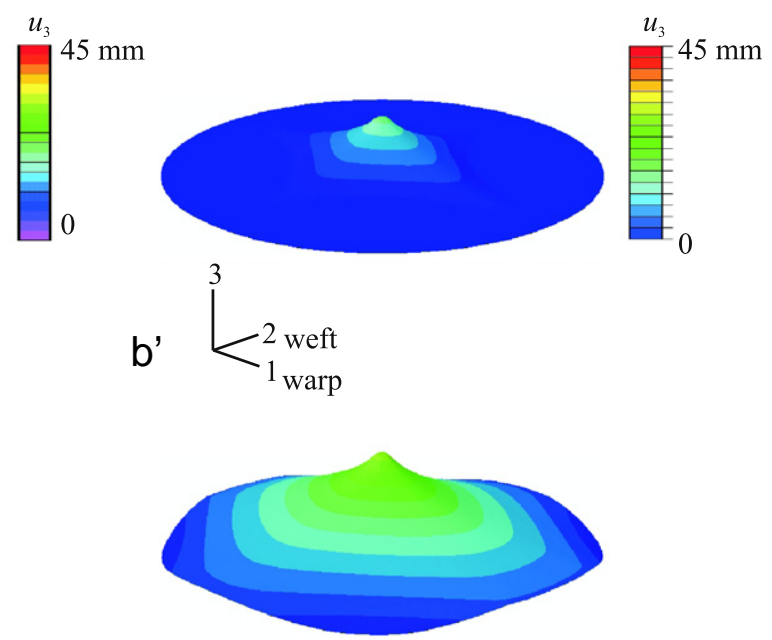

$c^{\prime}$

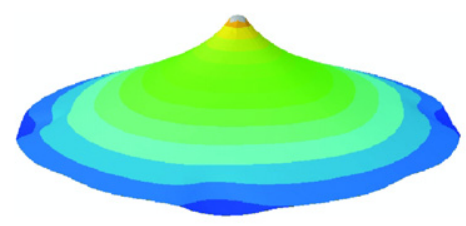

d'

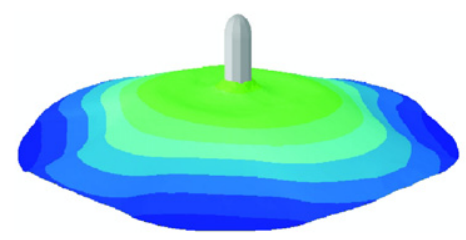

Fig. 20. Contours of transverse displacement for experiment (left) and simulation (right) for circular cut-out with $v_{i}=30 \mathrm{~m} / \mathrm{s}$ at time after impact: (a), (a') $0.5 \mathrm{~ms}$; (b), (b') $1.0 \mathrm{~ms}$; (c), (c') $1.425 \mathrm{~ms}$; (d), (d') $1.575 \mathrm{~ms}$.

with an analytical rigid surface. The grip areas of the specimen were clamped between two rigid surfaces with a pressure of $480 \mathrm{kPa}$. The clamping pressure was determined from the amount that the fabric slipped from the clamps during the experiments. The interactions between the projectile and the fabric and the clamps and the fabric were modeled with the kinematic predictor/corrector (hard) contact algorithm. Contact between the clamps and the fabric was modeled with Coulombic friction with $\mu=0.3$, and contact between the projectile and the fabric was approximated as frictionless. A preload of $2 \mathrm{~N}$ was applied in the warp direction by specifying initial changes in crimp amplitude, $\Delta A_{1}^{0}$ and $\Delta A_{2}^{0}$, as well as initial deformation gradient, $F^{\mathrm{pre}}$. The preload procedure is described further in Appendix C.2. In these simulations, the failure criterion was set at $T_{k}^{\max }=122.5 \mathrm{~N}$ in order to match the ballistic limit measured in the experiments. This failure criterion is very close to the average load per yarn at which the warp strips failed in uniaxial tension (Fig. 8a). The time of computation on a PC with $3 \mathrm{~GB}$ of RAM and a single Xeon ${ }^{\mathbb{R}} 3.2 \mathrm{GHz}$ CPU (4 MB L2 cache, $1066 \mathrm{MHz}$ front-side bus speed) was under $30 \mathrm{~min}$. 
a

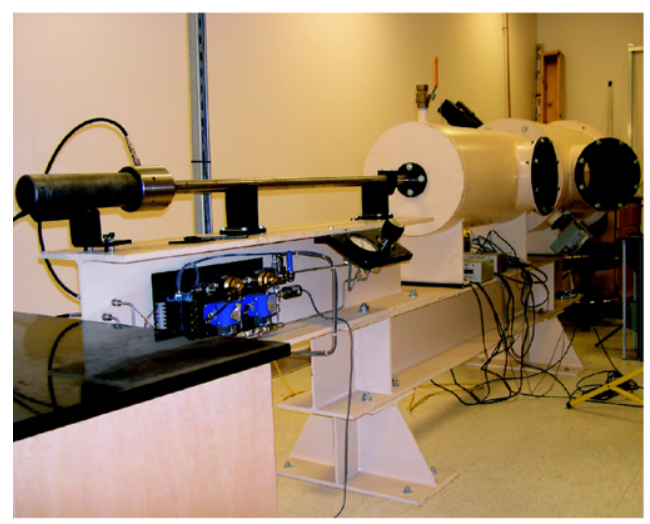

b

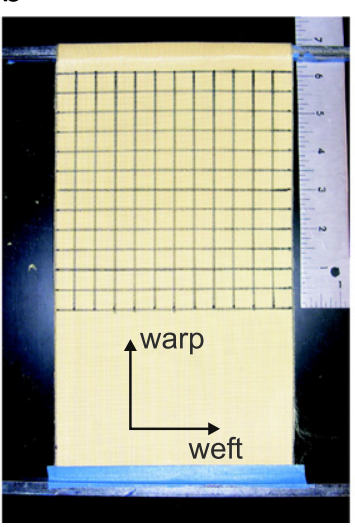

C

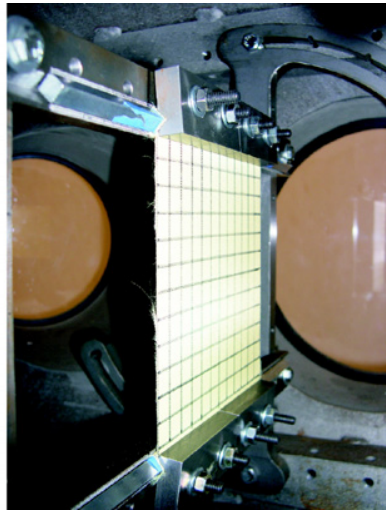

Fig. 21. Ballistic impact experiments at the ISN: (a) gas gun and closed chamber with viewing ports; (b) fabric was wrapped around steel bars; (c) fabric wrapped bars were clamped to the frame inside the chamber.

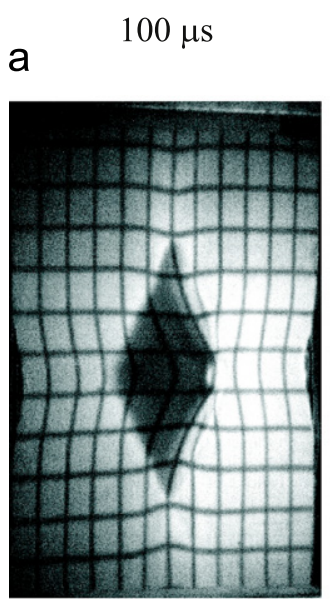

a'

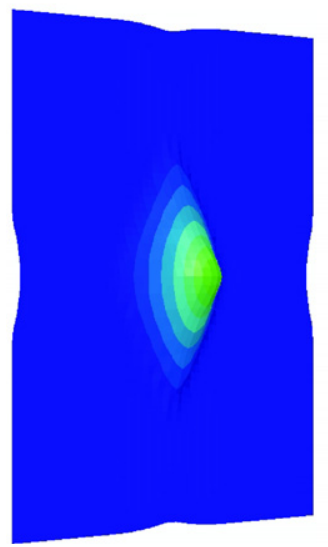

b

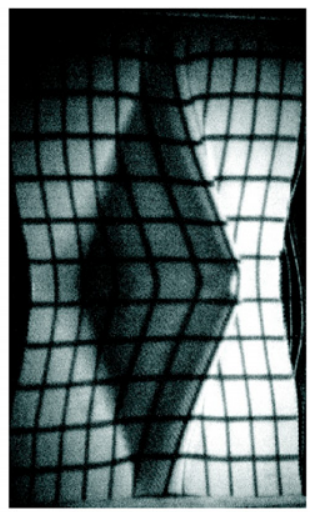

b'

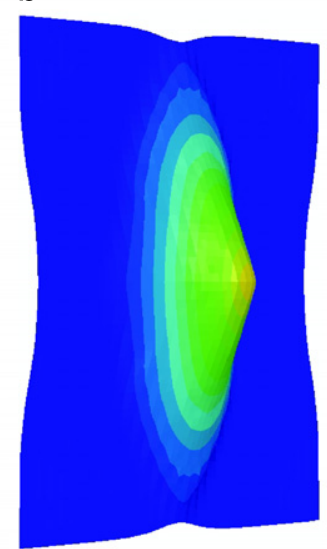

$300 \mu \mathrm{s}$

C

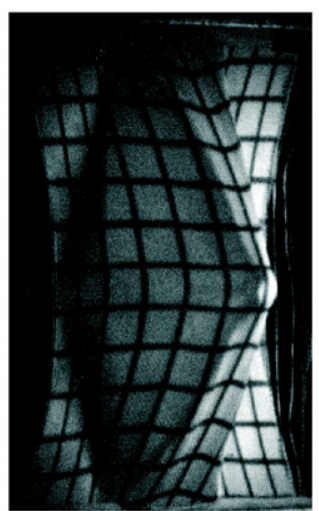

c'

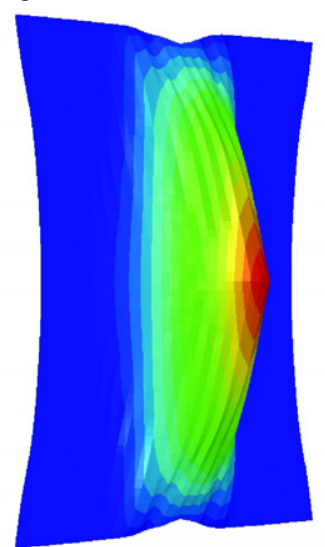

d

$400 \mu \mathrm{s}$

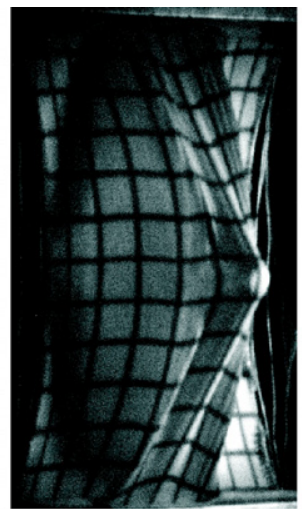

d'

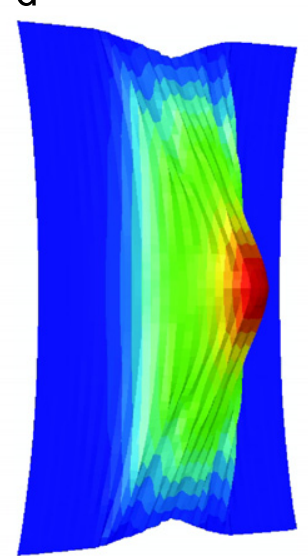

warp

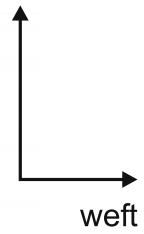

$u_{3}$

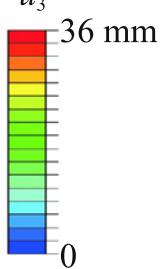

Fig. 22. Images of ISN experiment (top) and simulated contours of transverse displacement (bottom) for $v_{\mathrm{i}}=171 \mathrm{~m} / \mathrm{s}$ at time after impact: (a), (a') $100 \mu \mathrm{s}$; (b), (b') $200 \mu \mathrm{s}$; (c), (c') $300 \mu \mathrm{s}$; (d), (d') $400 \mu \mathrm{s}$.

\subsection{Results of ballistic impact experiments and simulations}

The fabric finite elements accurately predict the defeat of projectiles with initial velocity $v_{\mathrm{i}} \approx 170 \mathrm{~m} / \mathrm{s}$. Fig. 22 shows experiment and simulation with $v_{\mathrm{i}}=171 \mathrm{~m} / \mathrm{s}$. At $t=100 \mu \mathrm{s}$ (Fig. 22a, a'), transverse displacement waves are propagating in the warp and weft directions away from the point of impact. The longitudinal strain waves have already reached the free 
and clamped edges of the fabric, setting the primary yarns in motion toward the projectile. The primary yarns drag orthogonal secondary yarns toward the projectile as well, causing the fabric to bow in toward its center. The longitudinal strain waves have caused the fabric to slip from the clamps. In the experiment, at the sides of the specimen, several warp yarns have unraveled due to yarn slip. At $t=200 \mu$ s (Fig. 22b, $\mathrm{b}^{\prime}$ ), the transverse displacement waves in the warp direction reach the clamps and are reflected. The transverse displacement waves travel faster in the warp direction than in the weft direction because the strains are much higher in the warp yarns than in the weft yarns. By $t=300 \mu$ s, the transverse deformation has spread horizontally almost to the edges of the specimen (Fig. $22 \mathrm{c}, \mathrm{c}^{\prime}$ ), and, at $t=400 \mu \mathrm{s}$, the fabric stops the projectile (Fig. 22d, $\mathrm{d}^{\prime}$ ). The simulation qualitatively predicts all features of the deformation with the exception of the small amount of unraveling that occurs at the edges of the fabric. Quantitatively, the simulation predicts within approximately $10 \%$ the transverse displacement at the point of impact (Fig. 23). A similar experiment was conducted with the camera repositioned for a profile view of the fabric. Using the same gas, chamber pressures, and diaphragms as in the previous experiment resulted in a slightly lower projectile velocity of $v_{\mathrm{i}}=164 \mathrm{~m} / \mathrm{s}$. Transverse displacement profiles along the center impacted warp yarn were measured manually from the images. A simulation with $v_{\mathrm{i}}=164 \mathrm{~m} / \mathrm{s}$ underpredicts by up to $10 \%$ the displacement at the projectile, but otherwise the displacement profiles are very similar (Fig. 24).

In the experiments, the residual velocity of the projectile, $v_{\mathrm{r}}$, exhibits three distinct regimes (Fig. 25): (1) below the ballistic limit, $v_{\mathrm{i}} \approx 200 \mathrm{~m} / \mathrm{s}$, the projectile does not penetrate the fabric; (2) at intermediate velocities, $v_{\mathrm{i}} \approx 200-275 \mathrm{~m} / \mathrm{s}$, the residual velocity is approximately $60 \%$ of the initial velocity; ( 3 ) at high velocities, $v_{\mathrm{i}}>275 \mathrm{~m} / \mathrm{s}$, there is a jump in the residual velocity to approximately $90 \%$ of the initial velocity. Plotting the results in terms of energy in Fig. 26 , we see that the energy absorbed by the fabric, $E_{\mathrm{abs}}=\frac{1}{2} m_{\mathrm{proj}}\left(v_{\mathrm{i}}^{2}-v_{\mathrm{r}}^{2}\right)$, reaches a maximum within the intermediate velocity regime and then, on average, declines through the high velocity regime. Shim et al. (1995), Tan et al. (2003), and Lim et al. (2003) observed the same regimes in their experiments. The noise in the residual velocity data at high velocities has numerous

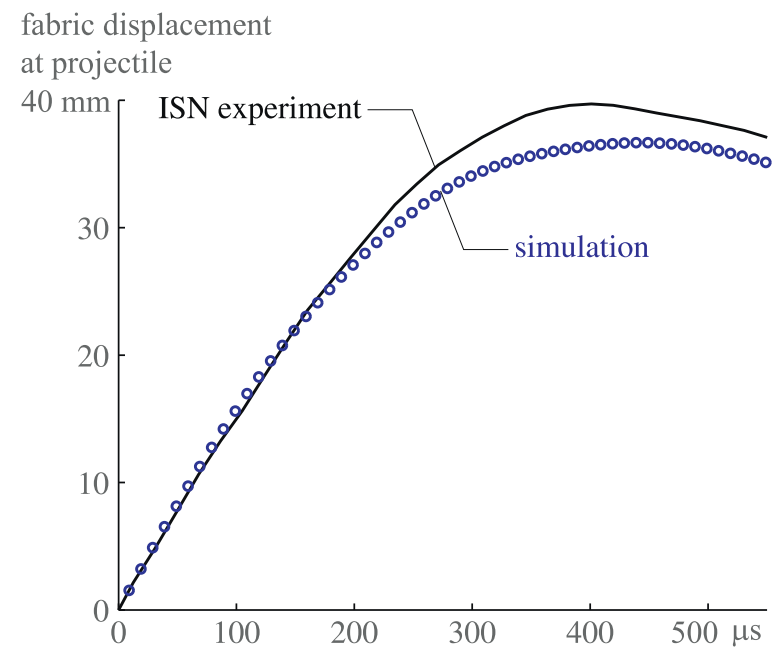

Fig. 23. Prediction of transverse displacement at point of impact for $v_{\mathrm{i}}=171 \mathrm{~m} / \mathrm{s}$.

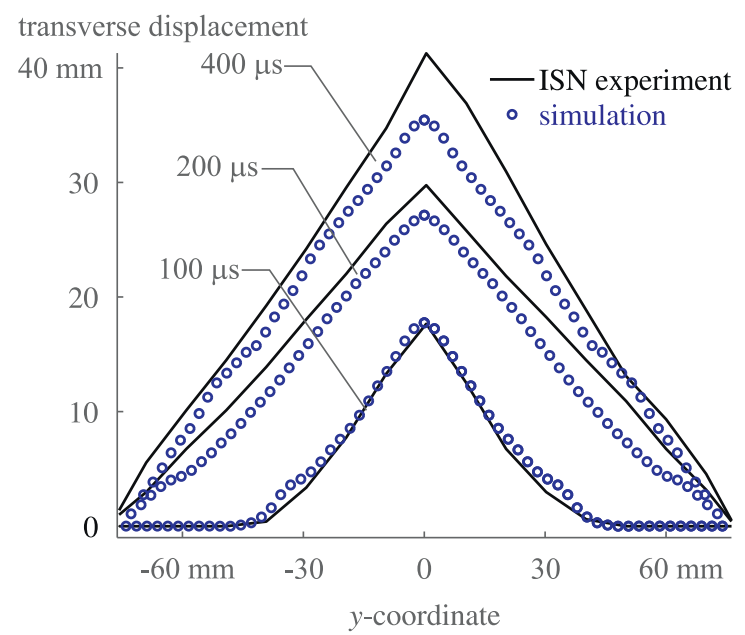

Fig. 24. Prediction of transverse displacement profiles through point of impact for $v_{\mathrm{i}}=164 \mathrm{~m} / \mathrm{s}$. 

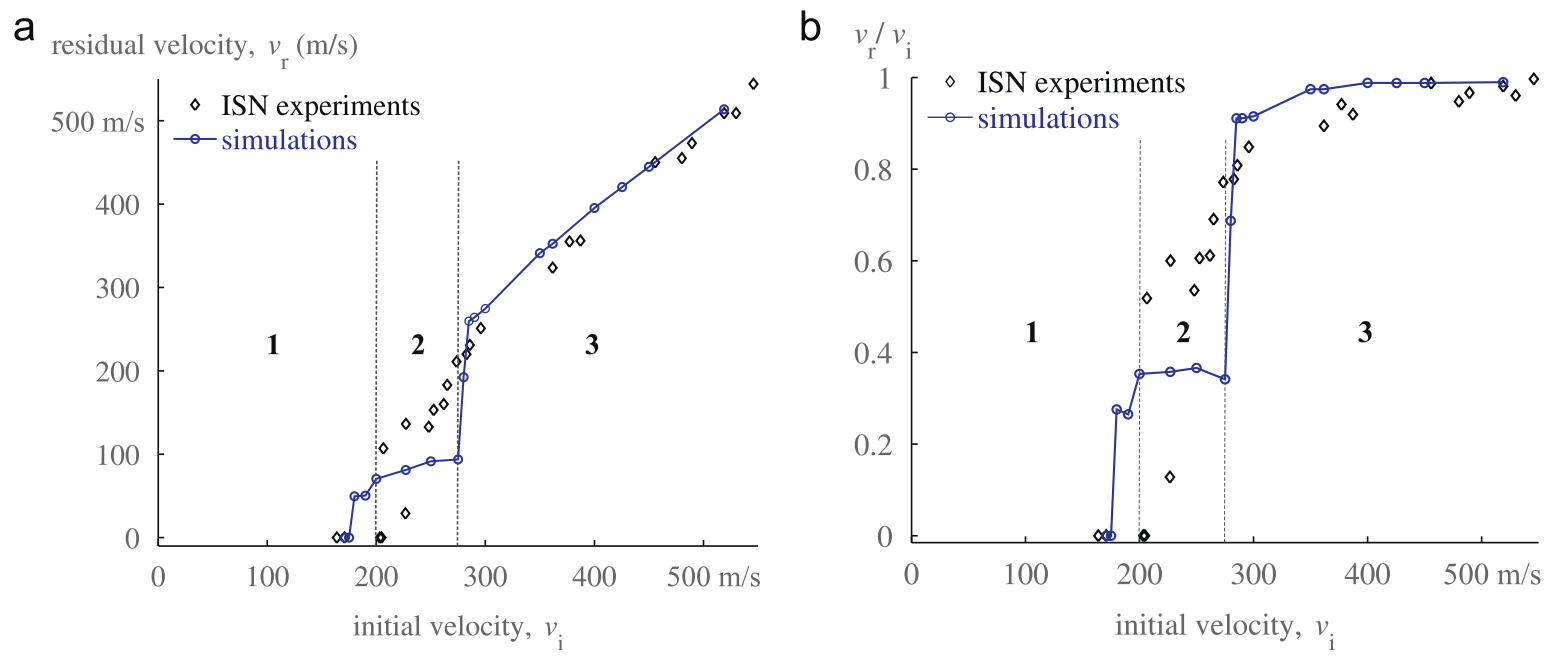

Fig. 25. Residual velocity of the projectile $\left(v_{\mathrm{r}}\right)$ in ballistic impact simulations and experiments: (a) residual velocity versus initial velocity, (b) residual velocity as a fraction of initial velocity versus initial velocity.

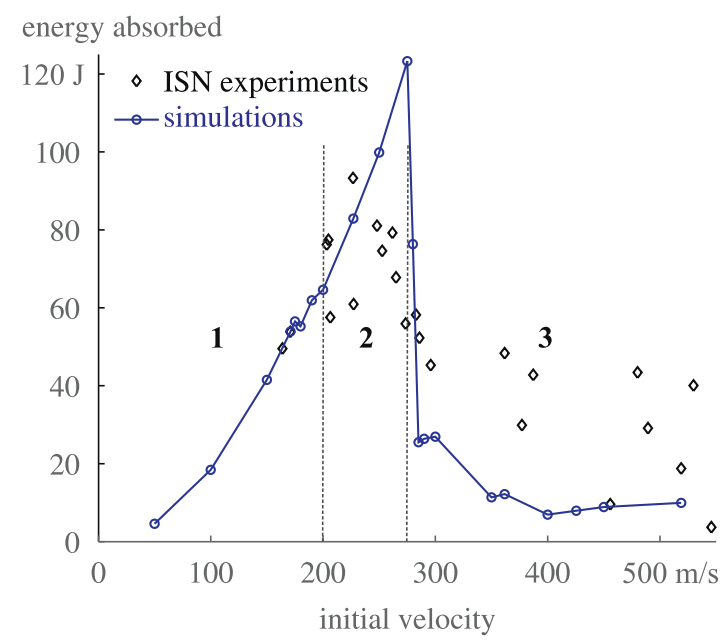

Fig. 26. Energy absorbed by the fabric as a function of the initial velocity of the projectile.

potential sources, including variations in the properties of the fabric, the clamping pressure, the preload of the fabric, the angle of incidence of the projectile, and the exit direction of the projectile.

The simulations predict the existence of the three regimes and, at intermediate velocities, the energy absorbed by the fabric (Figs. 25 and 26). However, the simulations significantly underpredict the energy absorbed in the high velocity regime.

A much larger portion of the fabric deforms prior to failure in the intermediate velocity regime than in the high velocity regime. Fig. 27 illustrates a typical intermediate velocity impact event. When the projectile first impacts the fabric at $v_{\mathrm{i}}=227 \mathrm{~m} / \mathrm{s}$, the tension in the primary warp yarns quickly increases as longitudinal strain waves propagate through the fabric away from the projectile in either direction (Fig. 27a'). After the strain waves reach the clamps, the yarn tension increases only at the projectile until about $t=100 \mu \mathrm{s}$ (Fig. 27b'). Over the next approximately $100 \mu \mathrm{s}$, the yarn tension at the projectile decreases by half, and the tension in the primary warp yarns becomes uniform again. The decrease in yarn tension is due to the slip of the fabric from the clamps; if slip is not allowed in the simulation, the tension plateaus but does not decrease. Once the transverse displacement wave reaches the clamps (Fig. 27c, $c^{\prime}$ ), the yarn tension begins to increase rapidly throughout the primary warp yarns until failure occurs (Fig. 27d, $\mathrm{d}^{\prime}$ ). The tension in the primary yarns is then relieved (Fig. 27e'). By this time, nearly the entire specimen has deformed, and the majority of the energy of the projectile has been transformed into the motion and strain of the yarns. Fig. 28 illustrates a typical high velocity impact event. With $v_{\mathrm{i}}=362 \mathrm{~m} / \mathrm{s}$, the yarns break well before the transverse displacement wave reaches the clamps. As a result, in the high velocity regime, less of the fabric deforms and far less energy is absorbed than in the intermediate velocity regime.

What separates the intermediate velocity and high velocity regimes is whether failure occurs during the first or second increase in warp yarn tension. Fig. 29 plots the history of warp yarn tension at the projectile, which is generally the maximum 


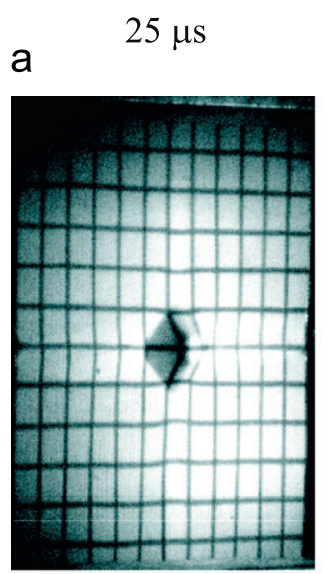

b $\quad 100 \mu \mathrm{s}$

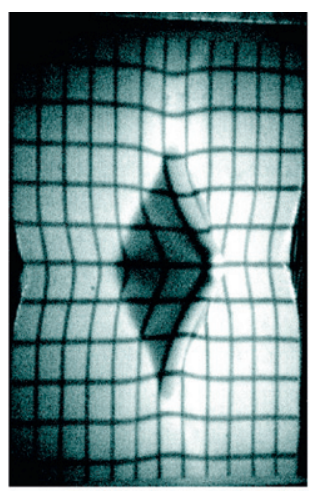

$a^{\prime}$
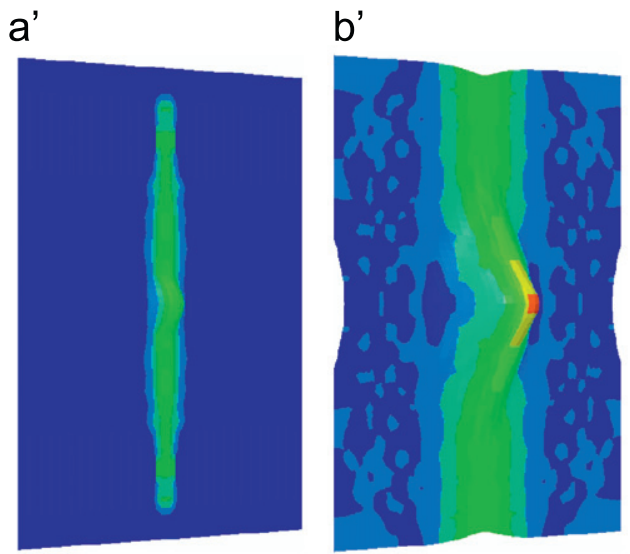

$200 \mu \mathrm{s}$

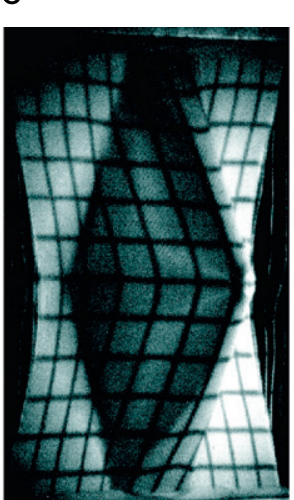

c'

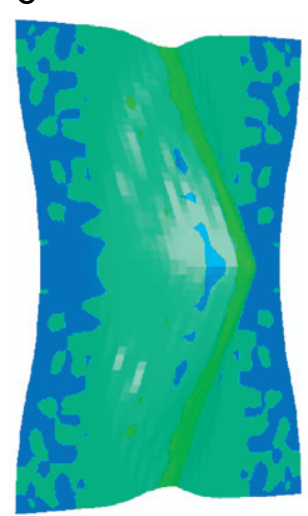

$250 \mu \mathrm{s}$

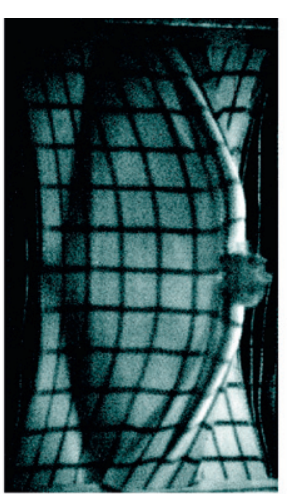

$300 \mu \mathrm{s}$

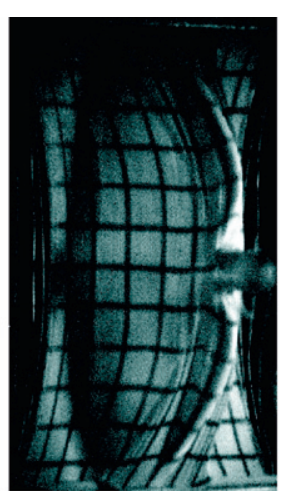

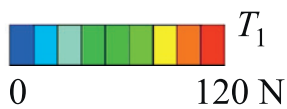

d'

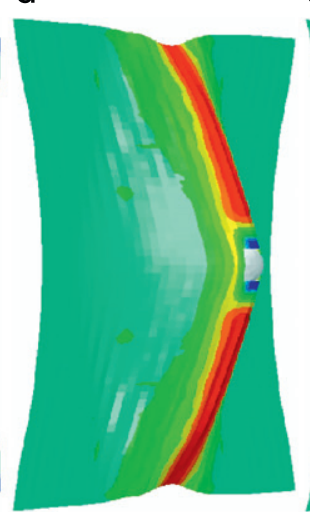

e'

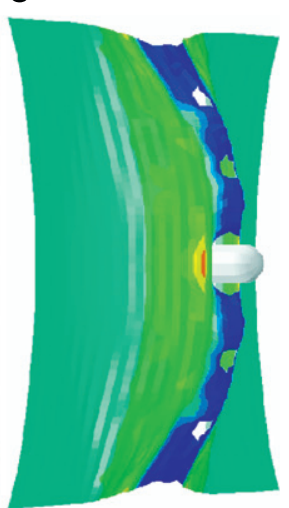

0

$120 \mathrm{~N}$

Fig. 27. Images of ISN experiment (top) and simulated contours of warp yarn tension (bottom) for $v_{\mathrm{i}}=227 \mathrm{~m} / \mathrm{s}$ at time after impact: (a), (a') $25 \mu \mathrm{s}$; (b), ( $\mathrm{b}^{\prime}$ ) $100 \mu \mathrm{s} ;(\mathrm{c}),\left(\mathrm{c}^{\prime}\right) 200 \mu \mathrm{s} ;(\mathrm{d}),\left(\mathrm{d}^{\prime}\right) 250 \mu \mathrm{s} ;(\mathrm{e}),\left(\mathrm{e}^{\prime}\right) 300 \mu \mathrm{s}$.

tension in the fabric, for four simulations, including the three simulations just presented. During the first increase in tension, the tension and the rate of increase in tension are proportional to the initial velocity of the projectile. In the high velocity regime, the first increase in tension is severe enough for the yarns to break. In the intermediate velocity regime, on the other hand, the yarns survive the first increase in tension. After peaking, the tension decreases until the transverse deformation of the fabric reaches the clamps. Then, the tension begins to increase again, eventually causing the yarns to break. Thus, at intermediate projectile velocities, the fabric has time to deform extensively, absorbing considerable energy in the process. Shim et al. observed similar histories of yarn tension in simulations of the ballistic impact of a Twaron ${ }^{\circledR}$ fabric.

In the high velocity regime, the simulations predict failure prematurely, and therefore underpredict the deformation of the fabric and the amount of energy absorbed, because yarn slip has not yet been incorporated into the fabric finite elements. Yarn slip occurs when the gradient in tension along a yarn is large enough to overcome the friction between the yarn and its crossing yarns. During the first increase in warp yarn tension, large tension gradients develop around the projectile (see Figs. $27 b^{\prime}$ and $28 a^{\prime}$ ) causing yarn slip to occur. In simulations of ballistic impact with each yarn explicitly modeled, Duan et al. (2005, 2006a), Zeng et al. (2006), and Grujicic et al. (2008a) showed that yarn slip itself does not absorb significant energy. However, yarn slip does improve ballistic performance. Analogous to yielding in metals or polymers, it blunts the tension concentration at the projectile, increasing the time to failure of the fabric. In the high velocity regime, yarn slip therefore indirectly increases the energy absorption capacity of the fabric. These results agree with the simulations of Zeng et al., which predict the ballistic limit of a Twaron fabric to decrease if there is too much friction between yarns, and the experiments of Lastnik and Karageorgis (1982), which show the ballistic limit of a Kevlar fabric-reinforced composite to decrease with decreasing yarn mobility. At intermediate velocities, in contrast, yarn slip plays almost no role for, during the second increase in tension, these severe tension gradients do not develop (Fig. $\left.27 \mathrm{c}^{\prime}, \mathrm{d}^{\prime}\right)$. The slip-free assumption of the simulations is most likely also the reason that the failure criterion needed to be set higher for this set of simulations than for the simulations of the low velocity experiments. 

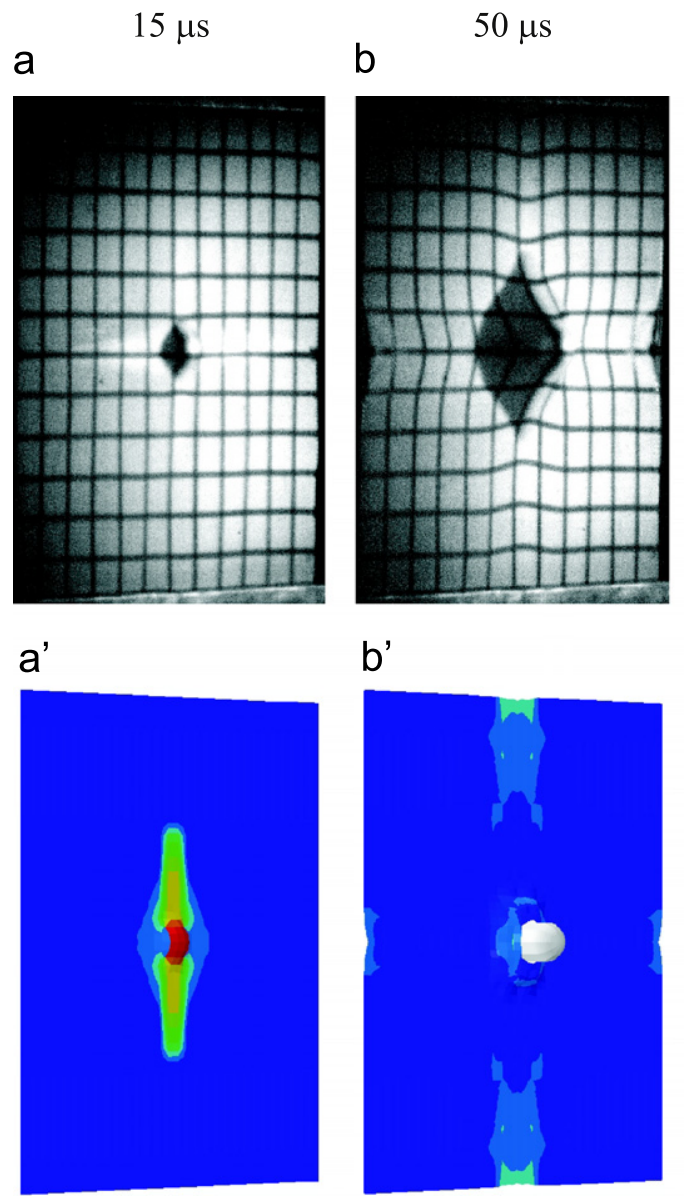

b

b'

$50 \mu \mathrm{s}$

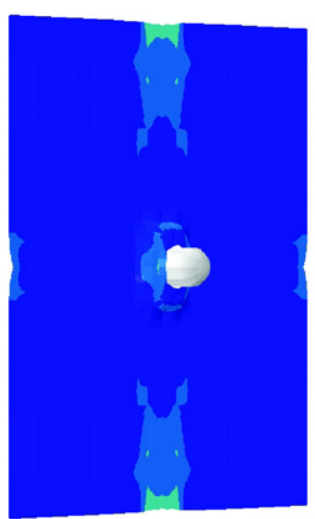

$85 \mu \mathrm{s}$

C

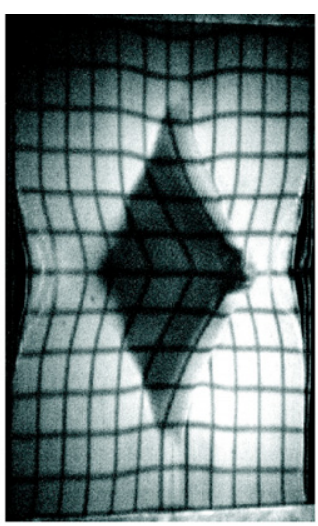

$c^{\prime}$

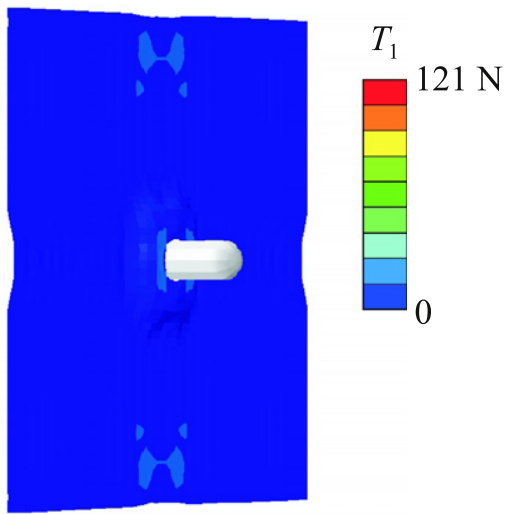

Fig. 28. Images of ISN experiment (top) and simulated contours of warp yarn tension (bottom) for $v_{\mathrm{i}}=362 \mathrm{~m} / \mathrm{s}$ at time after impact: (a), (a') $15 \mu \mathrm{s}$; (b), (b') $50 \mu \mathrm{s} ;(\mathrm{c}),\left(\mathrm{c}^{\prime}\right) 85 \mu \mathrm{s}$.

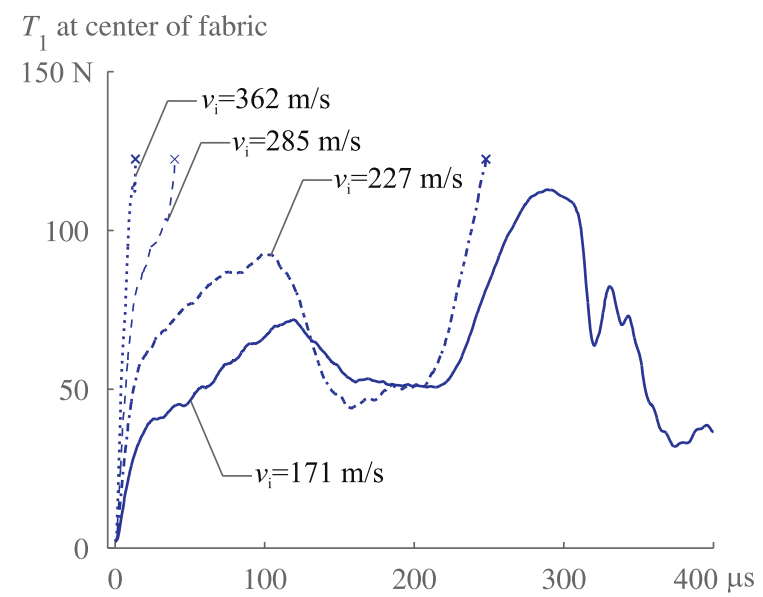

Fig. 29. Simulated warp yarn tension at the center of the fabric: at high projectile velocities, the fabric fails during the first increase in tension; at intermediate projectile velocities, the fabric fails during the second increase in tension.

\section{Conclusion}

The woven fabric finite elements accurately and efficiently simulate a variety of quasi-static tension tests and projectile impact experiments. They successfully simulate the response to arbitrary loadings because their macroscopic predictions derive from the deformation of a unit cell which faithfully represents the mesostructure and modes of deformation of the 
fabric. The elements predict not only macroscopic deformation but also the yarn-level quantities necessary for anticipating failure and designing composite forming processes, for example. Because they model the fabric as a continuum, not discrete yarns, the elements are computationally inexpensive and therefore capable of simulating real-sized, complex structures, including multi-ply systems. Within the elements, we use the novel approach of defining nodal degrees of freedom with physically correct mass to represent the change in crimp amplitude of the yarns. Solving for the crimp amplitudes in this manner automatically includes the inertia of uncrimping and is much more efficient than the energy minimization procedure or numerical methods used in other woven fabric simulations of this type.

The structure of the fabric finite elements is versatile and may be applied to many existing or proposed materials. Other plain-weave fabrics composed of rate-independent yarns may be simulated simply by adjusting the entirely physical material parameters. Viscoelastic yarn deformation may be included in the elements by modifying the constitutive relations for yarn extension and transverse compression. Fabrics of different weave patterns, such as twill, basket, or satin weaves, would require unit cells with more yarns and corresponding additional internal equilibrium arguments and degrees of freedom, but the methods for determining the deformed geometry of the unit cell and calculating the continuum-level stress would not change. Furthermore, owing to their continuum nature, the elements may be integrated seamlessly with other material models for the simulation of woven fabric-reinforced composites. The elements may also be extended to simulate relative yarn slip by introducing two additional nodal degrees of freedom, one for the slip of each yarn family. Although typically not a significant source of energy dissipation during ballistic impact, yarn slip is important at very high projectile velocities because it reduces the tension concentration at the projectile, enabling the fabric to survive longer. Finite elements with relative yarn slip enabled will be presented in a future publication.

\section{Acknowledgments}

This research was supported by the U.S. Army Research Office through the MIT Institute for Soldier Nanotechnologies under contract number W911NF-07-D-0004. We thank Professor R. Radovitzky for providing the explicit finite element framework, Adlib, in which we originally implemented the fabric model.

\section{Appendix A. Verification of membrane element approximation}

In simulations of a quasi-static indentation test of length scale similar to that of the ballistic impact experiments, meshes with membrane elements with the constitutive response of King et al. (2005) and shell elements with the constitutive response of King and Socrate (2005) showed nearly the same resistance to deformation (Fig. 30). The fabric was a single layer of Kevlar KM2, $20 \mathrm{~cm} \times 20 \mathrm{~cm}$, clamped on all sides. The simulations were conducted with Abaqus/ Standard. Eight-node full integration membrane elements and four-node reduced integration shell elements with hourglass control from the Abaqus/Standard library were used.

\section{Appendix B. Effect in simulations of fabric clamping pressure on the response of the fabric}

The deformation of the fabric depends strongly on the amount of slip of the fabric from the frame (Fig. 31). The slip from the frame is controlled by the clamping pressure and the coefficient of friction between the clamping surfaces and the

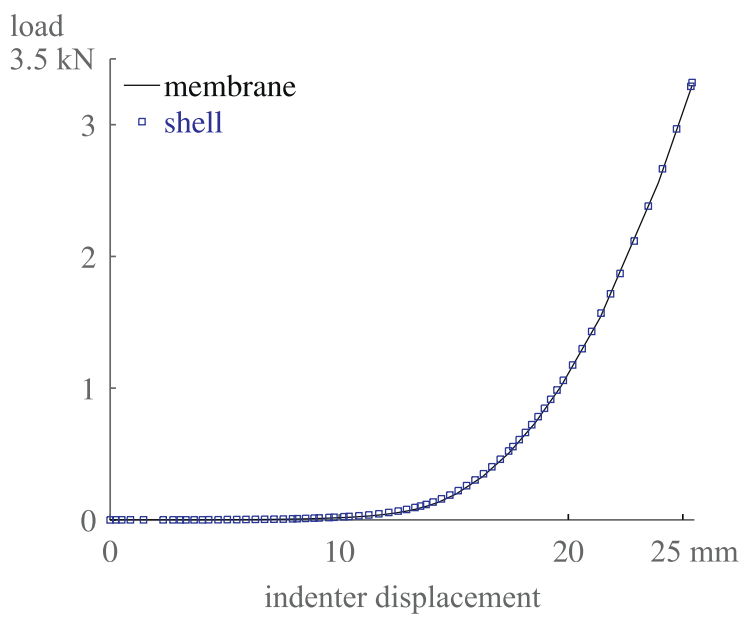

Fig. 30. Meshes with membrane and shell elements show nearly the same resistance to deformation in a quasi-static indentation simulation. 
a

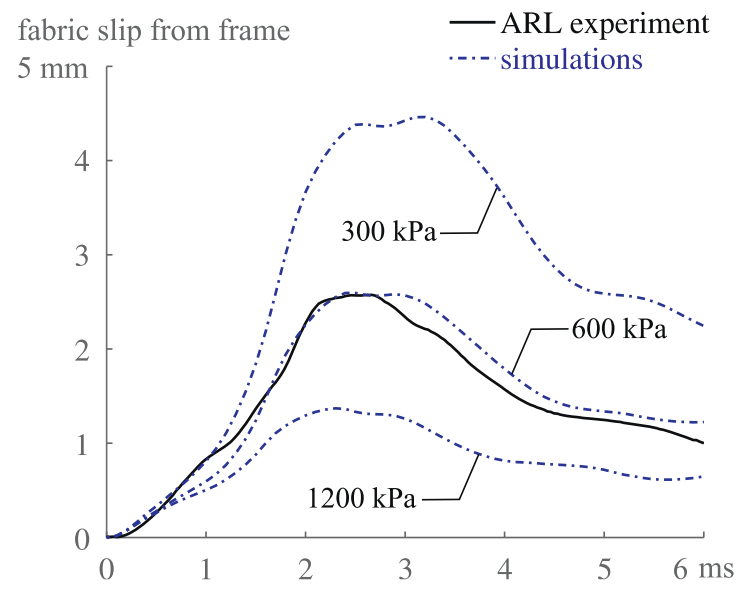

b

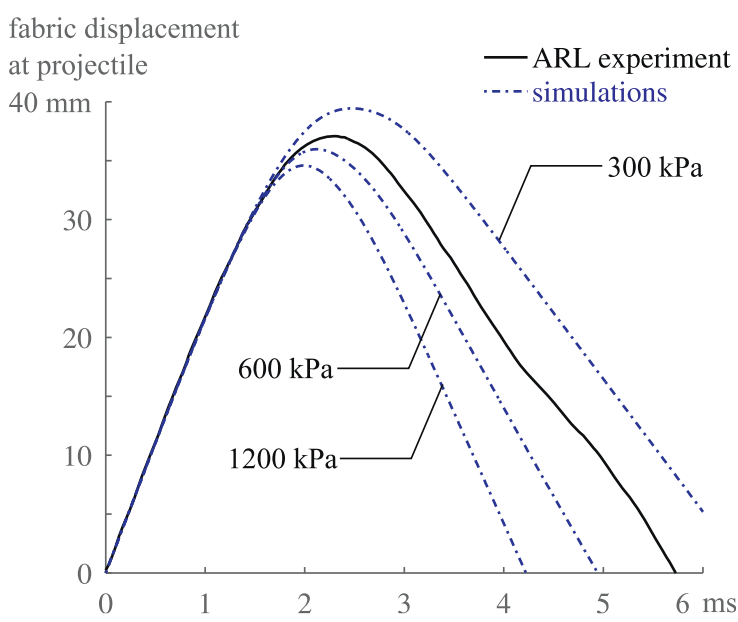

Fig. 31. Effect of fabric clamping pressure on simulation of circular cut-out ARL experiment with $v_{\mathrm{i}}=22 \mathrm{~m} / \mathrm{s}$ : (a) average in-plane slip displacement of the fabric from the frame increases with decreasing clamping pressure, (b) transverse displacement at point of impact increases with decreasing clamping pressure.

fabric. In the simulation of the ARL experiments, the coefficient of friction was assumed to be $\mu=0.3$. The clamping pressure in the simulations was varied until the simulated slip from the frame matched the experimental slip from the frame. We illustrate in Fig. 31a how the clamping pressure was determined to be $600 \mathrm{kPA}$ for the circular cut-out. A similar procedure was used for the square cut-out.

\section{Appendix C. Determining preload parameters in simulations of projectile impact experiments}

A preload was applied to the fabric in the simulations of both the low velocity impact experiments and the ballistic impact experiments by specifying an initial deformation gradient, $\mathbf{F}^{\text {pre }}$, and initial changes in yarn crimp amplitude, $\Delta A_{1}^{0}$ and $\Delta A_{2}^{0}$. The deformation gradient used to determine the deformed geometry of the fabric unit cell in Eq. (7) becomes $\mathbf{F}^{\prime}=\mathbf{F F}^{\text {pre }}$. Alternatively, to the same end, $\mathbf{F}^{\text {pre }}$ could have been applied macroscopically to the fabric.

\section{C.1. Preload in simulations of low velocity impact experiments}

In the simulations of the low velocity (ARL) projectile impact experiments, a biaxial preload was applied parallel to the yarns of the fabric. In order to determine the preload parameters, various biaxial yarn tensions were applied to a single 4-node, reduced integration finite element. For each set of yarn tensions, $\mathbf{F}^{\mathrm{pre}}, \Delta A_{1}^{0}$ and $\Delta A_{2}^{0}$ were tabulated. A parametric study with preload parameters corresponding to varying yarn tensions was then conducted with the circular cut-out simulation. We found that a preload of $1 \mathrm{~N}$ per yarn in the warp and weft directions provided a good fit to the experimental data during the initial stage of deformation, before the transverse displacement wave reaches the clamps. This yarn tension corresponds to initial changes in yarn crimp amplitude, $\Delta A_{1}^{0}=-0.0093 \mathrm{~mm}$ and $\Delta A_{2}^{0}=-0.0238 \mathrm{~mm}$, and non-zero relevant components of the initial deformation gradient $F_{11}^{\mathrm{pre}}=1.0040$ and $F_{22}^{\mathrm{pre}}=1.0135$. An identical preload was used in the simulation of the experiment with the square cut-out.

\section{C.2. Preload in simulations of ballistic impact experiments}

The preload of the fabric in the simulations of the ballistic impact (ISN) experiments was determined by a parametric study similar to that for the low velocity case. Since, in the high velocity experiments, only the warp yarns were clamped, the warp tension alone was varied. The appropriate preload was found to be $2 \mathrm{~N}$ per yarn in the warp direction, resulting in initial changes in yarn crimp amplitude, $\Delta A_{1}^{0}=-0.0215 \mathrm{~mm}$ and $\Delta A_{2}^{0}=0.0024 \mathrm{~mm}$, as well as non-zero relevant components of the initial deformation gradient, $F_{11}^{\mathrm{pre}}=0.9984$ and $F_{22}^{\mathrm{pre}}=1.0081$.

\section{References}

Boisse, P., Borr, M., Buet, K., Cherouat, A., 1997. Finite element simulations of textile composite forming including the biaxial fabric behaviour. Compos. B $28,453-464$

Boisse, P., Gasser, A., Hivet, G., 2001. Analyses of fabric tensile behaviour: determination of the biaxial tension-strain surfaces and their use in forming simulations. Compos. A 32, 1395-1414. 
Boisse, P., Zouari, B., Daniel, J., 2006. Importance of in-plane shear rigidity in finite element analyses of woven fabric composite preforming. Compos. A 37, 2201-2212.

Boisse, P., Zouari, B., Gasser, A., 2005. A mesoscopic approach for the simulation of woven fibre composite forming. Compos. Sci. Technol. 65, 429-436.

Cao, J., Akkerman, R., Boisse, P., Chen, J., Cheng, H.S., de Graaf, E.F., Gorczyca, J.L., Harrison, P., Hivet, G., Launay, J., Lee, W., Liu, L., Lomov, S.V., Long, A., de Luycker, E., Morestin, F., Padvoiskis, J., Peng, X.Q., Sherwood, J., Stoilova, T., Tao, X.M., Verpoest, I., Willems, A., Wiggers, J., Yu, T.X., Zhu, B., 2008. Characterization of mechanical behavior of woven fabrics: experimental methods and benchmark results. Compos. A 39 (6), $1037-1053$.

Cheng, M., Chen, W.M., Weerasooriya, T., 2005. Mechanical properties of Kevlar ${ }^{\circledR}$ KM2 single fiber. J. Eng. Mater. Technol. 127 (2), $197-203$.

Cunniff, P.M., 1992. An analysis of the system effects in woven fabrics under ballistic impact. Text. Res. J. 62, 495-509.

Cunniff, P.M., 1996. A semiempirical model for the ballistic impact performance of textile-based personnel armor. Text. Res. J. 66 (1), 45-59.

Duan, Y., Keefe, M., Bogetti, T.A., Cheeseman, B.A., 2005. Modeling friction effects on the ballistic impact behavior of a single-ply high-strength fabric. Int. J. Imp. Eng. 31 (8), 996-1012.

Duan, Y., Keefe, M., Bogetti, T.A., Cheeseman, B.A., Powers, B., 2006a. A numerical investigation of the influence of friction on energy absorption by a high-strength fabric subjected to ballistic impact. Int. J. Imp. Eng. 32 (8), 1299-1312.

Duan, Y., Keefe, M., Bogetti, T.A., Powers, B., 2006b. Finite element modeling of transverse impact on a ballistic fabric. Int. J. Mech. Sci. 48, 33-43.

Grujicic, M., Arakere, G., He, T., Gogulapati, M., Cheeseman, B.A., 2008a. A numerical investigation of the influence of yarn-level finite-element model on energy absorption by a flexible-fabric armour during ballistic impact. Proc. Inst. Mech. Eng. Part L J. Mat. Des. App. 222 (L4), $259-276$.

Grujicic, M., Bell, W.C., He, T., Cheeseman, B.A., 2008b. Development and verification of a meso-scale based dynamic material model for plain-woven single-ply ballistic fabric. J. Mater. Sci. 43 (18), 6301-6323.

Hamila, N., Boisse, P., Sabourin, F., Brunet, M., 2009. A semi-discrete shell finite element for textile composite reinforcement forming simulation. Int. J. Numer. Methods Eng. 79, 1443-1466.

Ivanov, I., Tabiei, A., 2004. Loosely woven fabric model with viscoelastic crimped fibres for ballistic impact simulations. Int. J. Numer. Methods Eng. 61 (10), 1565-1583.

Kawabata, S., Niwa, M., Kawai, H., 1973. The finite deformation theory of plain weave fabrics. Part I: the biaxial deformation theory. J. Text. Inst. 64, 21-46.

King, M.J., 2006. A continuum constitutive model for the mechanical behavior of woven fabrics including slip and failure. Ph.D. Thesis, Massachusetts Institute of Technology, Cambridge, MA, USA.

King, M.J., Jearanaisilawong, P., Socrate, S., 2005. A continuum constitutive model for the mechanical behavior of woven fabrics. Int. J. Solids Struct. 42, 3867-3896.

King, M.J., Socrate, S., 2005. A continuum anisotropic shell formulation to model the three-dimensional deformation response of woven fabrics. In: Proceedings of the IASS/IACM 5th International Conference on the Computation of Shells and Spatial Structures.

Lastnik, A.L., Karageorgis, C., 1982. The effect of resin concentration and laminating pressures on Kevlar ${ }^{\circledR}$ fabric bonded with a modified phenolic resin. Technical Report, US Army Natick Research \& Development Center.

Lim, C.T., Shim, V.P.W., Ng, Y.H., 2003. Finite element modeling of the ballistic impact of fabric armor. Int. J. Imp. Eng. 28, 13-31

Martínez, M.A., Navarro, C., Cortés, R., Rodríguez, J., 1993. Friction and wear behaviour of Kevlar fabrics. J. Mater. Sci. 28, $1305-1311$.

Parga-Landa, B., Hernández-Olivares, F., 1995. An analytical model to predict impact behavior of soft armors. Int. J. Imp. Eng. 16 (3), $455-466$.

Peng, X.Q., Cao, J., 2005. A continuum mechanics-based non-orthogonal constitutive model for woven composite fabrics. Compos. A 36, 859-874.

Phoenix, S.L., Porwal, P.K., 2003. A new membrane model for the ballistic impact response and v-50 performance of multiply fibrous systems. Int. J. Solids Struct. 40 (24), 6723-6765.

Realff, M.L., Boyce, M.C., Backer, S., 1997. A micromechanical model of the tensile behavior of woven fabric. Text. Res. J. 67, 445-459.

Roylance, D., 1980. Stress wave propagation in fibres: effect of crossovers. Fibre Sci. Technol. 13, $385-395$.

Roylance, D., Chammas, P., Ting, J., Chi, H., Scott, B., 1995. Numerical modeling of fabric impact. In: Proceedings of the National Meeting of the ASME.

Roylance, D., Wang, S.S., 1978. Penetration mechanics of textile structures: influence of non-linear viscoelastic relaxation. Polym. Eng. Sci. 18, 1068-1072.

Roylance, D., Wilde, A., Tocci, G., 1973. Ballistic impact of textile structures. Text. Res. J. 43, 34-41.

Shahkarami, A., Vaziri, R., 2007. A continuum shell finite element model for impact simulation of woven fabrics. Int. J. Imp. Eng. 34, 104-119.

Shim, V.P.W., Tan, V.B.C., Tay, T.E., 1995. Modelling deformation and damage characteristics of woven fabric under small projectile impact. Int. J. Imp. Eng. $16,585-605$

Shockey, D.A., Erlich, D.C., Simons, J.W., 1999. Lightweight fragment barriers for commercial aircraft. In: 18th International Symposium on Ballistics, San Antonio, TX, pp. 1192-1199.

Smith, J.C., McCrackin, F.L., Schiefer, H.F., Stone, W.K., Towne, K.M., 1956. Stress-strain relationships in yarns subjected to rapid impact loading: 4. Transverse impact tests. J. Res. Natl. Bur. Stand. 57, 83-89.

Talebi, H., Wong, S.V., Hamouda, A.M.S., 2009. Finite element evaluation of projectile nose angle effects in ballistic perforation of high strength fabric. Compos. Struct. 87 (4), 314-320.

Tan, V.B.C., Ching. T.W., 2006. Computational simulation of fabric armour subjected to ballistic impacts. Int. J. Imp. Eng. 32, 1737-1751.

Tan, V.B.C., Lim, C.T., Cheong, C.H., 2003. Perforation of high-strength fabric by projectiles of different geometry. Int. J. Imp. Eng. 28, 207-222.

Tan, V.B.C., Shim, V.P.W., Zeng, X., 2005. Modelling crimp in woven fabrics subjected to ballistic impact. Int. J. Imp. Eng. 32 (1-4), $561-574$.

Tanov, R.R., Brueggert, M., 2003. Finite element modeling of non-orthogonal loosely woven fabrics in advanced occupant restraint systems. Fin. Elem. Anal. Des. 39, 357-367.

Taylor, W.J., Vinson, J.R., 1990. Modeling ballistic impact into flexible materials. AIAA J. 28 (12), 2098-2103.

Termonia, Y., 2004. Impact resistance of woven fabrics. Text. Res. J. 74 (8), 723-729.

Ting, C., Ting, J., Cunniff, P., Roylance, D., 1998. Numerical characterization of the effects of transverse yarn interaction on textile ballistic response. In: International SAMPE Technical Conference Series, pp. 57-67.

Walsh, T.F., Lee, B.L., Song, J.W., 1998. Penetration failure of Spectra ${ }^{\circledR}$ polyethylene fiber-reinforced ballistic-grade composites. In: Impact Response and Dynamic Failure of Composites and Laminate Materials, Pts. 1 and 2, Key Engineering Materials, vol. 141-1. Trans Tech Publications, Switzerland, pp. 367-382.

Xue, P., Peng, X., Cao, J., 2003. A non-orthogonal constitutive model for characterizing woven composites. Compos. A 34, $183-193$.

Zeng, X.S., Shim, V.P.W., Tan, V.B.C., 2005. Influence of boundary conditions on the ballistic performance of high-strength fabric targets. Int. J. Imp. Eng. 32, 631-642.

Zeng, X.S., Tan, V.B.C., Shim, V.P.W., 2006. Modelling inter-yarn friction in woven fabric armour. Int. J. Numer. Methods Eng. 66 (8), 1309-1330.

Zhang, G.M., Batra, R.C., Zheng, J., 2008. Effect of frame size, frame type, and clamping pressure on the ballistic performance of soft body armor. Compos. B 39 (3), 476-489. 